\title{
Disturbance Observer Enhanced Neural Network LPV Control for a Blended-wing-body large Aircraft
}

\author{
Shiqian Liu, ${ }^{*}$ Member, IEEE, James F. Whidborne, Senior Member, IEEE, Sunan Chumalee
}

\begin{abstract}
The problem of trajectory tracking control for a Blended-Wing-Body (BWB) large aircraft with model parameter uncertainties and unknown disturbances is considered. A Linear Parameter-Varying (LPV) model is derived from the nonlinear dynamics of the BWB aircraft from which a robust linear parameter-varying controller is designed to track a desired trajectory. Using a Single Quadratic Lyapunov Function (SQLF) and an infinite number of linear matrix inequalities to be evaluated at all vertices, a pair of positive definite symmetric matrix solutions is determined via Lyapunov stability theory and linear matrix inequality technique. Furthermore, a disturbance-observer is designed to process the unknown disturbances. Considering the plant exists some model errors except for disturbances, a Radial Basis Function Neural Network (RBFNN) approximation is embedded into the SQLF LPV controller to improve tracking performances, and a composite disturbance-observer based Neural Network Single Quadratic Lyapunov Function (NNSQLF) controller can realize desired trajectory tracking of the linear parameter-varying system through regulating performance weighting functions. The closed-loop system of trajectory tracking control is proved to be asymptotically stable by using Lyapunov theory. Simulation results of forward flight speed and altitude tracking control of the BWB aircraft show that the proposed disturbance-observer based NNSQLF control can robustly stabilize the LPV system and precisely track the desired trajectory by comparing with conventional SQLF control and Parameter-Dependent Lyapunov Functions (PDLF) control, even in unknown exterior disturbances and model uncertainties.
\end{abstract}

KEY WORDS: BWB aircraft; linear parameter-varying system; disturbance observer; trajectory tracking; neural network

\section{INTRODUCTION}

Blended-Wing-Body (BWB) aircraft have attracted considerable interest for their potential in delivering significant improvements in carbon emissions performance as well as reduced noise, higher speed, longer flight range, and broader internal volume compared with traditional "tube-and-wing" configurations [1]. However, the longitudinal stability of the BWB aircraft decreases as the angle of attack increases, even at small angles of attack, i.e., the lift curve shows a nonlinear increase at small angles of

\footnotetext{
Authors' addresses: S.Q. Liu is with School of Aeronautics and Astronautics, Shanghai Jiaotong University, Shanghai, 200240, China, E-mail: liushiqian@sjtu.edu.cn. J.F. Whidborne, is with School of Aerospace, Transport and Manufacturing, Cranfield University, Cranfield, MK43 0AL, U.K. E-mail: i.f.whidborne@cranfield.ac.uk, S. Chumalee is with Research and Development Centre for Space and Aeronautical Science and Technology, Royal Thai Air Force, Bangkok, Thailand, E-mail: schumalee@ hotmail.com
} 
attack because of flow separation [2], and the nonlinear change of pitching moment easily make the BWB aircraft unstable due to the aerodynamic center to move forward. Meanwhile, as pitch arm of the BWB aircraft is shorter, pitch trim ability of the BWB aircraft is insufficient when climbing at low speed and high angle of attack. These make the BWB aircraft design and flight control become more challenge [3].

The increased aspect ratios and large wing spans of BWB aircraft means it is possible to equip more flaps and engines to improve the aircraft control capability. Castro analyzed the flying qualities of a BWB aircraft designed by Cranfield University [4] and proposed a stability augmentation controller for their improvement. Rahman and Whidborne [5] proposed augmenting the control surfaces with the propulsion system and developed an integration strategy for the blended wing body aircraft. Yann et al. [6] used non-smooth optimization techniques for the simultaneous control gains and control surface total span for the BWB aircraft under handling quality constraints. However, these considered the BWB aircraft as a linear time-invariant plant and then used traditional linear control methods to design the controllers.

The dynamics of BWB aircraft are nonlinear so small perturbation Linear Time Invariant (LTI) dynamic models vary following their operating conditions. Although conventional gain-scheduling techniques can be used to handle this nonlinear property in a local range, they come with no guarantees on the robustness, performance, or even nominal stability over the entire operating range of the system. An alternative approach is to use linear parameter-varying control to handle the nonlinearity and plant uncertainty, and this guarantees stability, robustness, and performance properties of the closed-loop system [7]. Algebraic manipulation methods such as Jacobian linearization [8], state transformation or function substitution [9], are normally used to derive an LPV model from the original nonlinear model. Zhao and Nagamune suggested a switching LPV control to deal with inexact measurement of scheduling parameters [10]. Gahinet et al. proposed a LMI-based test for the robust stability/performance of linear systems with affine and possibly time varying parameter uncertainty [11]. Sato used LPV gain scheduling control to suppress gust effect and validated by an in-flight simulator [12]. Baranyi proposed TP model transformation for Quasi-LPV [13]. Most of them are belongs to the LPV models.

LPV control is an extension of $H_{\infty}$ control. An LPV controller is designed with all possible operating points considered simultaneously, so the resulting controller gain is automatically scheduled as system dynamics varies. The closed-loop system theoretically guarantees stability and performance throughout the operating range of the system. Gain-scheduled $H_{\infty}$ control synthesis and analysis for the LPV plant is usually based on SQLFs [14] or a Parameter-Dependent Lyapunov function [15]. Using SQLFs, for both the affine LPV models and the polytopic models, a finite number of linear matrix inequalities (LMIs) need only be evaluated at all vertices while, for grid LPV models, an infinite number of LMIs have to be evaluated at all points over the entire parameter space to determine feasible solutions[16]. Thus for a grid LPV model case, the resulting gain-scheduled controller has high computational on-line complexity [16]. Meanwhile, the SQLF method is usually more conservative than the 
PDLF design when the parameters are time-invariant or slowly varying [15], but there are an infinite number of LMIs to be evaluated for PDLFs at all points over the entire space of parameters, so the resulting gain-scheduled controller requires more complex on-line computations[16,17].

Since operating aircraft are subject to unknown disturbances such as wind, gust, turbulence and environmental noises, a Disturbance Observer-Based Control (DOBC) approach is introduced in this paper to enhance the disturbance attenuation ability and robustness performance of the LPV control. The DOBC technique has been applied to the control of nonlinear systems and systems with unknown disturbances for three decades [18], whereby an observer is designed to estimate external disturbances or ignore nonlinear dynamics and then compensate for them. Sun et al. [19] proposed a Disturbance-Observer (DO) Based robust relative pose control for spacecraft rendezvous and proximity operations under input saturation. Liu and Chen [20] proposed a DOBC for disturbance attenuation and rejection for a fixed wing UAV with wind disturbances. Li et al. [21] provided detailed design methods of DOBC and applied them to control a hypersonic vehicle.

Motivated by the work of Castro [4], Chumalee and Whidborne [15] and Chen et al. [18], a Disturbance-Observer based Neural Network Single Quadratic Lyapunov Function (DO-NNSQLF) LPV control scheme is proposed here to robustly stabilize the LPV system of the BWB aircraft with unknown disturbances. This method compensates for bounded disturbances by DO based control and provides robustness to model parameter uncertainties. And this DO-NNSQLF design is adaptive for varying flight parameters such as airspeed and altitude.

The main contributions of this paper can be summarized the following: the LPV system derived from a nonlinear dynamics model of the BWB aircraft with unknown disturbances, is controlled using a DO-NNSQLF control design. And the DONNSQLF LPV design involves a DO based forward feedback control for active compensation of observable disturbances. The LPV system of the BWB aircraft, subject to model uncertainty and bounded disturbance, is also robust stabilized using the DONNSQLF control. The $L_{2}$ induced norm index is considered in DO-NNSQLF LPV design to process the bounded residual disturbances. The LPV system of the BWB aircraft, with unknown bounded disturbances, is stabilized by using the DONNSQLF LPV design. The desired speed and altitude trajectories are tracked by using this trajectory tracking controller.

The paper is organized as follows. Section II establishes an LPV dynamics model of a BWB aircraft. Section III proposes a DO-NNSQLF LPV design of the BWB aircraft. Section IV gives examples of trajectory tracking control based on DO-NNSQLF LPV control. Section V gives some conclusions.

\section{DYNAMICS MODELING OF THE BWB AIRCRAFT}




\section{A. The BWB aircraft dynamics modeling}

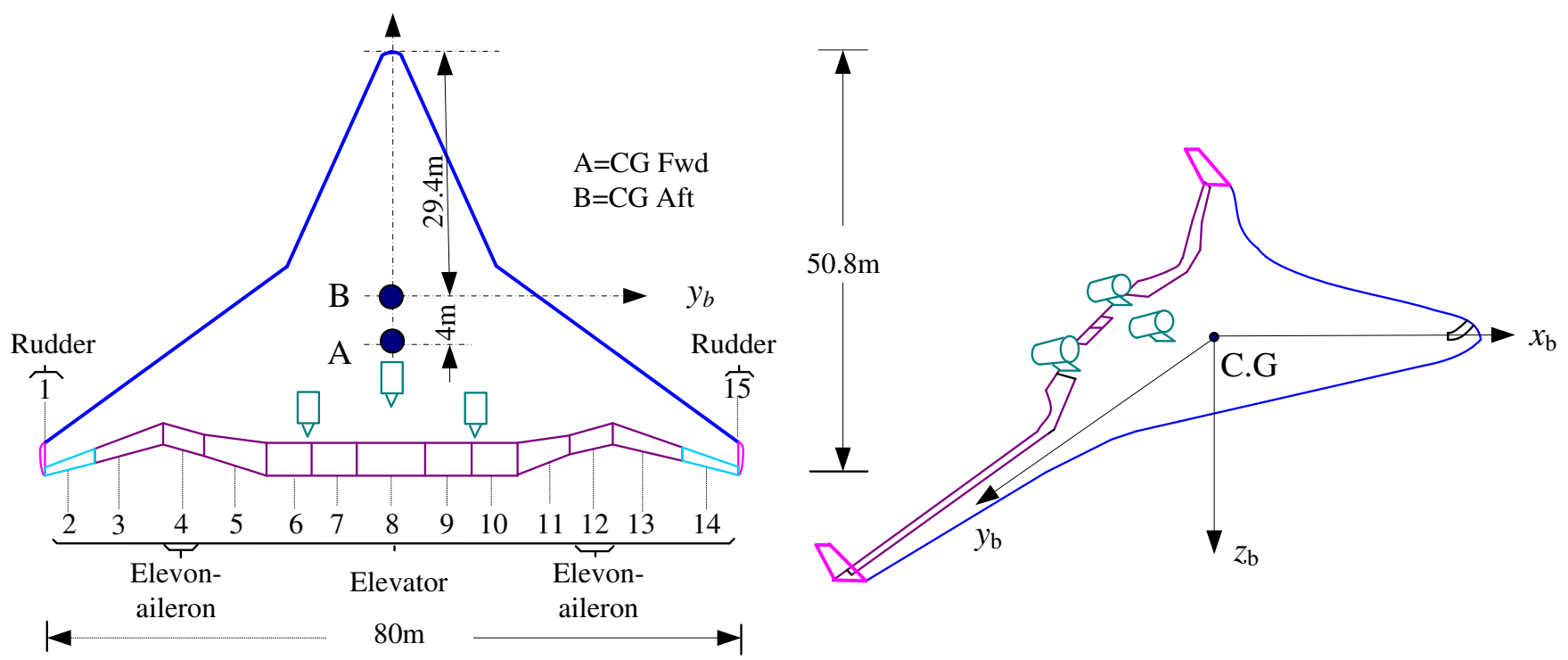

Fig.1 The BWB aircraft dynamics and structure geometry

The configuration of the BWB vehicle is as Fig.1. The considered model is a 50.8m length, 80m wing span BWB aircraft [4, 5], which is developed by Cranfield University for future airliner. Due to blended-wing-body configuration this aircraft has better aerodynamic and economical efficiency, and non-lift aerodynamic components are reduced. Hence, compared with conventional aircrafts, the lift-drag ratio of the BWB aircraft is enhanced and operating cost is decreased. It is feasible as the next generation of the advanced conventional aircraft. The structure parameters of the BWB aircraft are listed in Table 1.

Table 1 Parameters and coefficients for the studied BWB aircraft ${ }^{[4]}$

\begin{tabular}{cccccc}
\hline \hline Parameter & Value & Unit & Coefficient & Value & Unit \\
\hline$m$ & $3.7 \times 10^{5}$ & $\mathrm{~kg}$ & $T_{\delta \mathrm{a}}$ & 15 & $\mathrm{sec}$ \\
$\mathrm{S}$ & 841.7 & $\mathrm{~m}^{2}$ & $T_{\delta \mathrm{e}}$ & 15 & $\mathrm{sec}$ \\
$\bar{c}$ & 12.31 & $\mathrm{~m}$ & $T_{\delta \mathrm{r}}$ & 15 & $\mathrm{sec}$ \\
$\mathrm{b}$ & 80 & $\mathrm{~m}$ & $T_{\delta \mathrm{p}}$ & 1 & $\mathrm{sec}$ \\
$x_{G}$, & 30.4 & $\mathrm{~m}$ & $z_{G}$ & 0 & $\mathrm{~m}$ \\
$I_{\mathrm{xx}}$ & $4.703 \times 10^{7}$ & $\mathrm{~kg} \cdot \mathrm{m}^{2}$ & $I_{\mathrm{yy}}$ & $2.507 \times 10^{7}$ & $\mathrm{~kg} \cdot \mathrm{m}^{2}$ \\
$I_{\mathrm{zz}}$ & $9.973 \times 10^{7}$ & $\mathrm{~kg} \cdot \mathrm{m}^{2}$ & $I_{\mathrm{xz}}$ & 0.0 & $\mathrm{~kg} \cdot \mathrm{m}^{2}$ \\
\hline
\end{tabular}

The BWB vehicle is considered as a rigid body moving in air, which has fifteen flaps. Flap 1 and Flap 15 are the rudders, which are not in the wing. Flap 4 and Flap 12 are elevon-aileron, Flap 2 Flap 14 act as the elevators, see Fig.1.

The dynamics models of the BWB aircraft can be established and linearized using the Jacobian method about an equilibrium point, and a LTI model is obtained ${ }^{[4]}$. A number of equilibrium points can be defined for the BWB aircraft and, at each 
equilibrium point, some operating parameters are chosen as time-varying parameters $\rho$. When the time-varying parameters vary slowly, the linear time invariant model becomes an LPV model. And disturbances act on the BWB aircraft, which adversely affect the performance or stability of the flight control system. So a linearized parameter-varying dynamic model of the BWB aircraft with disturbances is established as follows

$$
\left\{\begin{array}{l}
\dot{x}(t)=A(\boldsymbol{\rho}) x(t)+B_{1}(\boldsymbol{\rho}) \omega(t)+\left(B_{2}(\boldsymbol{\rho}) u(t)+B_{3} d(t)\right) \\
z(t)=C_{1}(\boldsymbol{\rho}) x(t)+D_{11}(\boldsymbol{\rho}) \omega(t)+D_{12}(\boldsymbol{\rho}) u(t) \\
y(t)=C_{2}(\boldsymbol{\rho}) x(t)+D_{21}(\boldsymbol{\rho}) \omega(t)
\end{array}\right.
$$

where $x(t) \in \square^{p}$ is the state vector, $u(t) \in \square^{m_{2}}$ is the control input vector, $y(t) \in \square^{q_{2}}$ is the measurement output vector, the scheduled parameters $\rho=\left[\begin{array}{llll}\rho_{1}(t) & \rho_{2}(t) & \cdots & \rho_{n}(t)\end{array}\right]^{\mathrm{T}}$ is not known in advance, but can be measured in real time and lies in some set bounded by known minimum and maximum possible values, i.e., $\rho_{\mathrm{i}}(t) \in\left[\underline{\rho}_{\mathrm{i}}, \bar{\rho}_{\mathrm{i}}\right], \mathrm{i}=1,2, \ldots, \mathrm{n} . A(),. B_{2}(),. C_{i}($.$) , and D_{i j}($.$) , are$ known functions of time-varying parameters, and continuous mapping matrix functions. $d(t) \in \square^{m_{3}}$ denotes unknown bounded disturbance vector, $\omega(t) \in \square^{m_{1}}$ is the generalized disturbance vector, $z(t) \in \square^{q_{1}}$ is the controlled variable or error vector, continuous mapping matrix functions $A: \square^{n} \rightarrow \square^{p \times p}, B_{1}: \square^{n} \rightarrow \square{ }^{p \times m_{1}}, C_{1}: \square^{n} \rightarrow \square^{q_{1} \times p}, D_{11}: \square^{n} \rightarrow \square \square^{q_{1} \times m_{1}}, B_{3}$ is the disturbance effective matrix.

Assumption 1 [14]: Consider the LPV system (1), (i) $D_{22}=0$, (ii) $\left(B_{2}, C_{2}, D_{12}, D_{21}\right)$ are parameter independent (constant) matrices, and (iii) the pairs $\left(A(\rho), B_{2}\right)$ and $\left(A(\rho), C_{2}\right)$ are quadratically stabilizable and quadratically detectable over parameter space $\Theta$ respectively.

Note that Assumption 1- (i) can be overcome by a loop-shifting argument [22], and Assumption 1- (ii) is to reduce calculation overload due to infinite number of LMI constraints, which can be overcome by pre-filtering of the control inputs or post-filtering the measured outputs [14]. So the model (1) can be simplified as

$$
\left\{\begin{array}{l}
\dot{x}(t)=A(\rho) x(t)+B_{1}(\rho) \omega(t)+\left(B_{2} u(t)+B_{3} d(t)\right) \\
z(t)=C_{1}(\rho) x(t)+D_{11}(\rho) \omega(t)+D_{12} u(t) \\
y(t)=C_{2} x(t)+D_{21} \omega(t)
\end{array}\right.
$$

To match the disturbances from the control inputs, we set $B_{3}=B_{2}$, and suppose the disturbance is generated by a linear exogenous system,

$$
\left\{\begin{array}{l}
\dot{\zeta}(t)=W_{d} \varsigma(t) \\
d(t)=V_{d} \varsigma(t)
\end{array}\right.
$$

where $\varsigma \in \square^{p}, W_{\mathrm{d}}, V_{\mathrm{d}}$ are matrices with corresponding dimensions. For the LPV plant (2), design a dynamic output feedback LPV controller $K(\rho)$ as follows to meet the desired objective where the controller has the form 


$$
\left\{\begin{array}{l}
\dot{x}_{k}(t)=A_{k}(\boldsymbol{\rho}) x_{k}(t)+B_{k}(\boldsymbol{\rho}) y(t) \\
u(t)=C_{k}(\boldsymbol{\rho}) x_{k}(t)+D_{k}(\boldsymbol{\rho}) y(t)
\end{array}\right.
$$

and

$$
K(\boldsymbol{\rho})=\left(\begin{array}{ll}
A_{k}(\boldsymbol{\rho}) & B_{k}(\boldsymbol{\rho}) \\
C_{k}(\boldsymbol{\rho}) & D_{k}(\boldsymbol{\rho})
\end{array}\right)=\sum_{i=1}^{r} \alpha_{i} K_{i}, K_{i}=\left(\begin{array}{cc}
A_{k_{i}} & B_{k_{i}} \\
C_{k_{i}} & D_{k_{i}}
\end{array}\right), \quad \mathrm{i}=1,2, \ldots, \mathrm{r}
$$

For the LPV control design, first the observable disturbance $d(t)$ is neglected, then the closed-loop system of (2) and (4) is described by the state-space equations

$$
\left\{\begin{array}{l}
{\left[\begin{array}{c}
\dot{x}(t) \\
\dot{x}_{k}(t)
\end{array}\right]=A_{c l}(\rho)\left[\begin{array}{c}
x(t) \\
x_{k}(t)
\end{array}\right]+B_{c l}(\rho) \omega(t)} \\
z(t)=C_{c l}(\rho)\left[\begin{array}{c}
x(t) \\
x_{k}(t)
\end{array}\right]+D_{c l}(\rho) \omega(t)
\end{array}\right.
$$

where

$$
\begin{gathered}
A_{c l}(\rho)=\left[\begin{array}{cc}
A(\rho) & 0 \\
0 & 0_{p \times p}
\end{array}\right]+\bar{B} K(\rho) \bar{C}^{2} \sum_{i=1}^{r} \alpha_{i} \hat{A}_{c l_{i}}, \hat{A}_{c l_{i}}=\left[\begin{array}{cc}
\hat{A}_{i} & 0 \\
0 & 0_{p \times p}
\end{array}\right]+\bar{B} K_{i} \bar{C}, \\
B_{c l}(\rho)=\left[\begin{array}{c}
B_{1}(\rho) \\
0
\end{array}\right]+\bar{B} K(\rho) \bar{D}_{21}=\sum_{i=1}^{r} \alpha_{i} \hat{B}_{c l_{i}}, \hat{B}_{c l_{i}}=\left[\begin{array}{c}
\hat{B}_{1_{i}} \\
0
\end{array}\right]+\bar{B} K_{i} \bar{D}_{21}, \\
C_{c l}(\rho)=\left[\begin{array}{ll}
C_{1}(\rho) & 0
\end{array}\right]+\bar{D}_{12} K(\rho) \bar{C}=\sum_{i=1}^{r} \alpha_{i} \hat{C}_{c l_{i}}, \hat{C}_{c l_{i}}=\left[\begin{array}{ll}
\hat{C}_{1_{i}} & 0
\end{array}\right]+\bar{D}_{12} K_{i} \bar{C}, \\
D_{c l}(\rho)=D_{11}+\bar{D}_{12} K(\rho) \bar{D}_{21}=\sum_{i=1}^{r} \alpha_{i} \hat{D}_{c l_{i}}, \hat{D}_{c l_{i}}=D_{11_{i}}+\bar{D}_{12} K_{i} \bar{D}_{21} \\
\bar{B}=\left[\begin{array}{cc}
0 & B_{2} \\
I_{p} & 0
\end{array}\right], \bar{C}=\left[\begin{array}{cc}
0 & I_{p} \\
C_{2} & 0
\end{array}\right], \bar{D}_{12}=\left[\begin{array}{ll}
0 & D_{12}
\end{array}\right], \bar{D}_{21}=\left[\begin{array}{c}
0 \\
D_{21}
\end{array}\right] .
\end{gathered}
$$

\section{B Problem formulation}

To handle external disturbances and model parameter uncertainties with input constraints, we are now ready to state the robust control problem: Find a dynamic output feedback controller (4) combination with a disturbance observer of the LPV system (2) such that the following requirements are simultaneously satisfied:

(a) The closed-loop system, (2) and (4), is asymptotically stable with unknown observable disturbances $d(t)$;

(b) The closed-loop system ensures the induced $L_{2}$-norm of the operator mapping the disturbance signal $\omega(t)$ into the controlled signal $z(t)$ is bounded by $\gamma$, i.e., 


$$
\begin{gathered}
\|z(t)\|_{\ell_{2}}<\gamma\|\omega(t)\|_{2} \text { for all non-zero } \omega(t) \in \ell_{2}[0, \infty), \\
\|z(t)\|_{\ell_{2}}=\left[\int_{0}^{\infty} z^{T}(t) z(t) \mathrm{d} t\right]^{1 / 2}
\end{gathered}
$$

where $\gamma>0$ is the quadratic $H_{\infty}$ performance index.

\section{DO-SQLF DESIGN OF THE BWB aircraft}

\section{A. Control synthesis based on SQLF}

In this section, we shall derive sufficient conditions for the closed-loop system, (2) and (4), to satisfy the performance requirements of $(a)$ and $(b)$ in section II.B.

The system state matrix $A(\rho)$ in (2) can be written as a convex combination of the matrix vertices as

$$
A(\rho)=\operatorname{Co}\left\{\hat{A}_{1}, \hat{A}_{2}, \cdots, \hat{A}_{r}\right\}=\alpha_{1} \hat{A}_{1}+\alpha_{2} \hat{A}_{2}+\cdots+\alpha_{r} \hat{A}_{r}
$$

where $\mathrm{r}=2^{\mathrm{n}}$, and

$$
\left[\begin{array}{c}
\hat{A}_{1} \\
\hat{A}_{2} \\
\hat{A}_{3} \\
\vdots \\
\hat{A}_{r}
\end{array}\right]=\left[\begin{array}{cccccc}
1 & \underline{\rho}_{1} & \underline{\rho}_{2} & \cdots & \underline{\rho}_{n-1} & \underline{\rho}_{n} \\
1 & \underline{\rho}_{1} & \underline{\rho}_{2} & \cdots & \underline{\rho}_{n-1} & \bar{\rho}_{n} \\
1 & \underline{\rho}_{1} & \underline{\rho}_{2} & \cdots & \bar{\rho}_{n-1} & \bar{\rho}_{n} \\
\vdots & & & & & \vdots \\
1 & \bar{\rho}_{1} & \bar{\rho}_{2} & \cdots & \bar{\rho}_{n-1} & \bar{\rho}_{n}
\end{array}\right]\left[\begin{array}{c}
\tilde{A}_{0} \\
\tilde{A}_{2} \\
\vdots \\
\tilde{A}_{n}
\end{array}\right],
$$

Similarly, $B_{1}(\rho), C_{1}(\rho)$ and $D_{11}(\rho)$ in (2) can also be written as a convex combination of the matrix vertices as (14), i.e.,

$$
\left(\begin{array}{ccc}
A(\rho) & B_{1}(\rho) & B_{2} \\
C_{1}(\rho) & D_{11}(\rho) & D_{12} \\
C_{2} & D_{21} & 0
\end{array}\right)=\sum_{i=1}^{r} \alpha_{i}\left(\begin{array}{ccc}
\hat{A}_{i} & \hat{B}_{1_{i}} & B_{2} \\
\hat{C}_{1_{i}} & \hat{D}_{11_{i}} & D_{12} \\
C_{2} & D_{21} & 0
\end{array}\right)
$$

In order to compute $\alpha_{i}$, we first calculate the normalized co-ordinate,

$$
\alpha_{\rho_{i}}=\frac{\bar{\rho}_{i}-\rho_{i}(t)}{\bar{\rho}_{i}-\underline{\rho}_{i}}, i=1,2, \cdots, n
$$

Then, for each vertex, $\mathrm{j}=1,2, \ldots, \mathrm{r}$, the corresponding polytopic co-ordinates are calculated by[23]

$$
\alpha_{j}=\prod_{i=1}^{n} \tilde{\alpha}_{\rho_{i}}, \tilde{\alpha}_{\rho_{i}}=\left\{\begin{array}{ll}
\alpha_{\rho_{i}}, & \text { if } \underline{\rho}_{i} \text { is a co-ordinate of } \Theta_{j} \\
1-\alpha_{\rho_{i}}, & \text { if } \bar{\rho}_{i} \text { is a co-ordinate of } \Theta_{j}
\end{array},\right.
$$


Lemma 1 [24] Given a symmetric matrix polytope, $M(\rho) \in \square{ }^{p \times p}$, for which $M(\rho)=\sum_{i=1}^{r} \alpha_{i} M_{i}$, is a negative definite symmetric matrix for all possible parameter trajectories, where $\alpha_{i}$ is determined by (17)and $(18), M(\rho)<0, \forall \rho \in \Theta$, if and only if $M_{i}<0, i=1,2, \ldots, \mathrm{m}$.

The gain-scheduled output feedback $H_{\infty}$ control problem using SQLFs is to compute a dynamic LPV controller $K(\rho)$ as (4), which stabilizes the closed-loop system of (2) and (4), and minimizes the closed loop quadratic $H_{\infty}$ performance as (12) along all possible parameter trajectories, $\forall \rho \in \Theta$.

Based on the SQLFs $V(x)=x^{T} P x$, there is an LPV controller $K(\boldsymbol{\rho})$ that stabilizes the closed-loop system, (2) and (4), and ensures the $L_{2}$-induced norm of the operator mapping the disturbance signal into the controlled signal is bounded by along all possible parameter trajectories if and only if there exists $P=P^{T}$ such that

$$
P>0, \frac{d}{d t}\left(x^{T} P x\right)+z^{T} z-\gamma^{2} \omega^{T} \omega<0, \forall \rho \in \Theta
$$

Inequality (19) leads to the scaled bounded real lemma inequality [25]

$$
\left[\begin{array}{ccc}
A_{c l}^{T}(\rho) P+P A_{c l}(\rho) & P B_{c l}(\rho) & C_{c l}^{T}(\rho) \\
* & -\gamma I & D_{c l}^{T}(\rho) \\
* & * & -\gamma I
\end{array}\right]<0
$$

where * denotes a symmetric matrix element. Substitute (7) (11) into (20) yields

$$
\sum_{i=1}^{r} \alpha_{i}\left[\begin{array}{ccc}
\hat{A}_{c l_{i}}^{T} P+P \hat{A}_{c l_{i}} & P \hat{B}_{c l_{i}} & \hat{C}_{c l_{i}}^{T} \\
* & -\gamma I & \hat{D}_{c l_{i}}^{T} \\
* & * & -\gamma I
\end{array}\right]<0
$$

Inequality (21) can be rewritten as [26]

$$
\sum_{i=1}^{r} \alpha_{i}\left(\Psi_{c l_{i}}+\bar{Q}^{T} K_{i}^{T} \bar{P}_{c l}+\bar{P}_{c l}^{T} K_{i} \bar{Q}\right)<0
$$

where

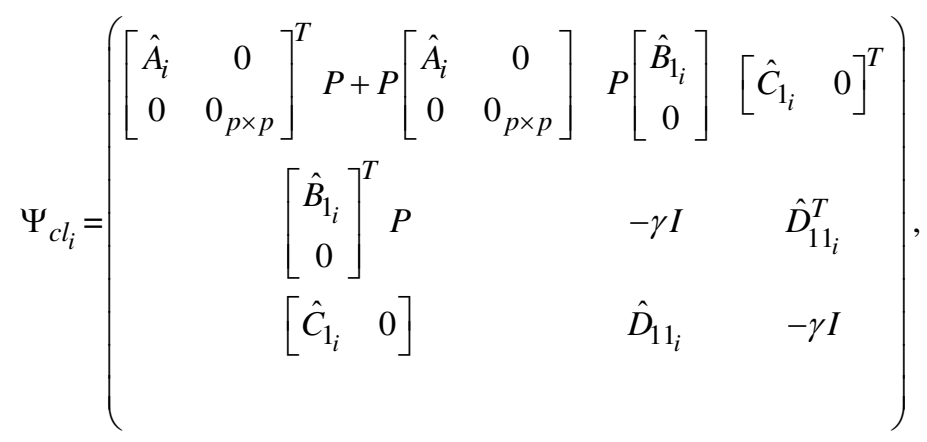




$$
\begin{aligned}
\bar{Q} & =\left[\begin{array}{lll}
\bar{C} & \bar{D}_{21} & 0_{\left(p+q_{2}\right) \times q_{1}}
\end{array}\right], \\
\bar{P}_{c l} & =\left[\begin{array}{lll}
\bar{B}^{T} P & 0_{\left(p+m_{2}\right) \times m_{1}} & \bar{D}_{12}^{T}
\end{array}\right] .
\end{aligned}
$$

To determine the quadratic Lyapunov variable $P$, a typical structure of $P$ can be selected as follows [15]

$$
P=\left[\begin{array}{cc}
X & -\left(X-Y^{-1}\right) \\
-\left(X-Y^{-1}\right) & X-Y^{-1}
\end{array}\right], P^{-1}=\left[\begin{array}{cc}
Y & Y \\
Y & \left(X-Y^{-1}\right) X Y
\end{array}\right],
$$

where positive definite symmetric matrix pair, $(X, Y) \in \square^{p \times p}$, meets $\left(X-Y^{-1}\right) \geq 0$, and $\operatorname{rank}\left(X-Y^{-1}\right) \leq p$.

Lemma 2 [27, Finsler's Lemma] Given an inequality problem of the form

$$
\Psi+Q^{T} K^{T} P+P K Q<0
$$

where $\Psi \in \square^{m \times m}$ is a symmetric matrix, $Q$ and $P$ are matrices with column dimension $m$. Let $\sigma$ be any real number, $\sigma \in \square$; the above problem is solvable for a matrix $K$ of compatible dimensions if and only if

$$
\left\{\begin{array}{c}
\Psi-\sigma Q^{T} Q<0 \\
\Psi-\sigma P^{T} P<0
\end{array}\right.
$$

By Lemma 2, LMIs (22) are solvable for $K_{\mathrm{i}}$ if and only if there exist a pair of positive definite symmetric matrices $(X, Y)$ satisfying the following LMIs:

$$
\begin{gathered}
\sum_{i=1}^{r} \alpha_{i}\left(\left(\begin{array}{cc}
N_{X} & 0 \\
0 & I
\end{array}\right)^{T}\left(\begin{array}{ccc}
\hat{A}_{i}^{T} X+X \hat{A}_{i} & X \hat{B}_{1_{i}} & \hat{C}_{1_{i}}^{T} \\
\hat{B}_{1_{i}}^{T} X & -\gamma I & \hat{D}_{11_{i}}^{T} \\
\hat{C}_{1_{i}} & \hat{D}_{11_{i}} & -\gamma I
\end{array}\right)\left(\begin{array}{cc}
N_{X} & 0 \\
0 & I
\end{array}\right)\right)<0 \\
\sum_{i=1}^{r} \alpha_{i}\left(\left(\begin{array}{cc}
N_{Y} & 0 \\
0 & I
\end{array}\right)^{T}\left(\begin{array}{ccc}
\hat{A}_{i}^{T} Y+Y \hat{A}_{i} & Y \hat{C}_{1_{i}}^{T} & \hat{B}_{1_{i}} \\
\hat{C}_{1_{i}} Y & -\gamma I & \hat{D}_{11_{i}} \\
\hat{B}_{1_{i}}^{T} & \hat{D}_{11_{i}}^{T} & -\gamma I
\end{array}\right)\left(\begin{array}{cc}
N_{Y} & 0 \\
0 & I
\end{array}\right)\right)<0 \\
\left(\begin{array}{cc}
X & I \\
I & Y
\end{array}\right)>0
\end{gathered}
$$

where $N_{X}$ and $N_{Y}$ denote bases of the null spaces of $\left[C_{2}, D_{21}\right]$ and $\left[B_{2}^{T}, D_{12}^{T}\right]$, respectively. Eq. (31) guarantees $X, Y>0$ and $\left(X-Y^{-1}\right) \geq 0$. By Lemma 1, (29) (31) need only be evaluated at all vertices. 


\section{B Disturbance-observer based control design}

For some applications, not only the precise system model is hard to obtain, but the uncertainty bound is hard to know in advance. This is obvious for flight control systems. The wind and turbulence occur at uncertain time and with unknown magnitudes. Hence a disturbance observer method is introduced into the LPV control to accommodate the large unknown external disturbances. A disturbance-observer based robust synthesized controller is proposed for the LPV system with system dynamic uncertainties and external disturbances. A disturbance observer to estimate and compensate for the disturbance in the control system Eq. (2), is given by [18] as

$$
\left\{\begin{array}{l}
\dot{z}_{d}=\left(W_{d}-L(x) B_{3} V_{d}\right) \hat{\zeta}-L(x)\left(A(\rho) x+B_{2} u\right) \\
\hat{\zeta}=z_{d}+L(x) x \\
\hat{d}=V_{d} \hat{\varsigma}
\end{array}\right.
$$

where $\hat{d}, L(x)$, and $z_{d}$ are estimated disturbance, the observer gains to be tuned for performance, and the internal state respectively. To prove the ability of the disturbance observer, the disturbance estimation error between the true values and estimated ones can be expressed as: $e_{d}=d-\hat{d}$, taking the derivative $\dot{e}_{d}$ along with Eqs.(2)-(4) and Eq.(32) gives

$$
\dot{e}_{\varsigma}(t)=\left(W_{d}-L(x) B_{3} V_{d}\right) e_{\varsigma}(t)-B_{1}(\rho) \omega
$$

where the term of $-B_{1}(\rho) \omega$ can be stabilized by the LPV controller. Provided that the disturbances vary slowly related to the observer dynamics, and the observer gain $L(x)$ is appropriately determined such that $W_{d}-L(x) B_{3} V_{d}$ is Hurwitz, then the Eq. (33) is shown that disturbance estimation error is asymptotically stable.

Remark 1 It can be seen that the error dynamics of Eq. (33) actually follows a linear form with a relatively simple structure. Moreover, it can be verified that the eigenvalues of the estimation error dynamics are constants or, more specifically, $\operatorname{eig}\left(W_{d}-L(x) B_{3} V_{d}\right)=\left\{-l_{\mathrm{i}}\right\}, i=1,2$ [20]. Therefore, by choosing the gain parameters $l_{i}$, the convergence rate of disturbance estimation can be intuitively adjusted regardless of state $x$.

We attempt to find an appropriate $L(x)$ to ensure that the disturbances can be significantly reduced or removed from the output channel finally. Thus the workload has been reduced by the LPV control.

Since the plant exists some model errors except for disturbances, such as actuator faults or model linearization error, a radial basis function neural network (RBFNN) is applied to approximate this unknown bounded model error. The architecture of the RBF-NN is presented in Fig.2, which includes three layers. 


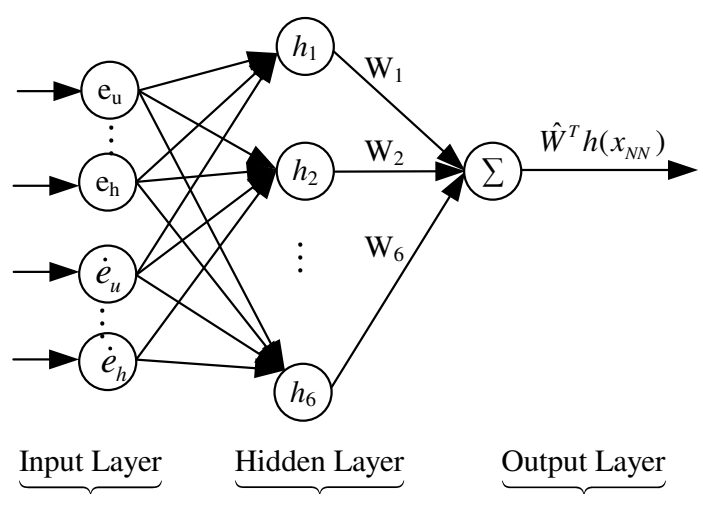

Fig.2. The architecture of the RBF-NN

First is the input layer, the net input is $x_{N N}=\left[\begin{array}{cc}e^{T} & \dot{e}^{T}\end{array}\right]^{T}$, where $e=x-x_{d}, \dot{e}=\dot{x}-\dot{x}_{d}, e=\left[\begin{array}{lllll}e_{u} & e_{w} & e_{q} & e_{\theta} & e_{h}\end{array}\right]^{T}, x_{\mathrm{d}}$ denotes the desired state variant. Second is the hidden layer, each node performs a membership function, and the output is $h\left(x_{N N}\right)=\left[h_{j}\left(x_{N N}\right)\right]^{T}, h\left(x_{N N}\right)$ is the basis function which can be selected as following Gaussian function

$$
h_{j}\left(x_{N N}\right)=\exp \left(\frac{-\left\|x_{N N}-c_{j}\right\|^{2}}{2 b_{j}^{2}}\right)
$$

where $c_{j}$ is the centre value of neural net $j, b_{\mathrm{j}}$ is the width value of the Gaussian function of net $j, j$ is the node number of the hidden layer. Third is the output layer. The output of the RBF-NN is

$$
y_{N N}=W^{T} h\left(x_{N N}\right)
$$

where $W$ is the best weight vector, whose update law is (114) in Appendix. The model error can be described as

$$
\Delta \Sigma=W^{* T} h\left(x_{N N}\right)+\varepsilon\left(x_{N N}\right)
$$

where $W^{*}$ denotes the optimal weight matrix of the RBF-NN, $\varepsilon$ is approximation error.

Based on the disturbance estimation from the DO and RBF model approximation, the composite control law with dynamic feedback (4) is designed as

$$
u(t)=u_{L P V}(t)+u_{d}(t)+u_{\mathrm{NN}}(t)
$$

where

$$
\begin{gathered}
u_{L P V}=u_{d} C_{k}(\rho) x_{k}(t)+D_{k}(\rho) y(t) \\
u_{d}=K_{d} \hat{d}=-B_{2}^{-1} B_{3} \hat{d}
\end{gathered}
$$

By using the condition of matching the input disturbances, substituting $B_{3}=B_{2}$ into Eq.(39) and yields 


$$
u_{d}=-B_{2}^{-1} B_{2} \hat{d}=-\hat{d}
$$

By using the RBF NN output of (35), the NN control input is

$$
u_{\mathrm{NN}}(t)=B_{2}^{-1} y_{N N}(t)=B_{2}^{-1} W^{T} h\left(x_{N N}\right)
$$

\section{A. Gain scheduled controller design based on NNSQLF}

This section gives the overview of the DO-LPV control for the BWB aircraft. The proposed controller structure is shown in Fig.3, the structure of the DO- LPV control design includes a DO, a RBF-NN, and a SQLF based LPV controller. The SQLF approach is used here to reduce the computation time in the nominal LPV control synthesis phase. The LPV controller is to realize the trajectory tracking, the DO is to observe the observable disturbance $d(t)$, and then the unobservable disturbance $\omega(t)$ effect is reduced by the LPV controller. The pre-filter is included to suppress high frequency signal inputs. The controller design is presented in detail as the next section.

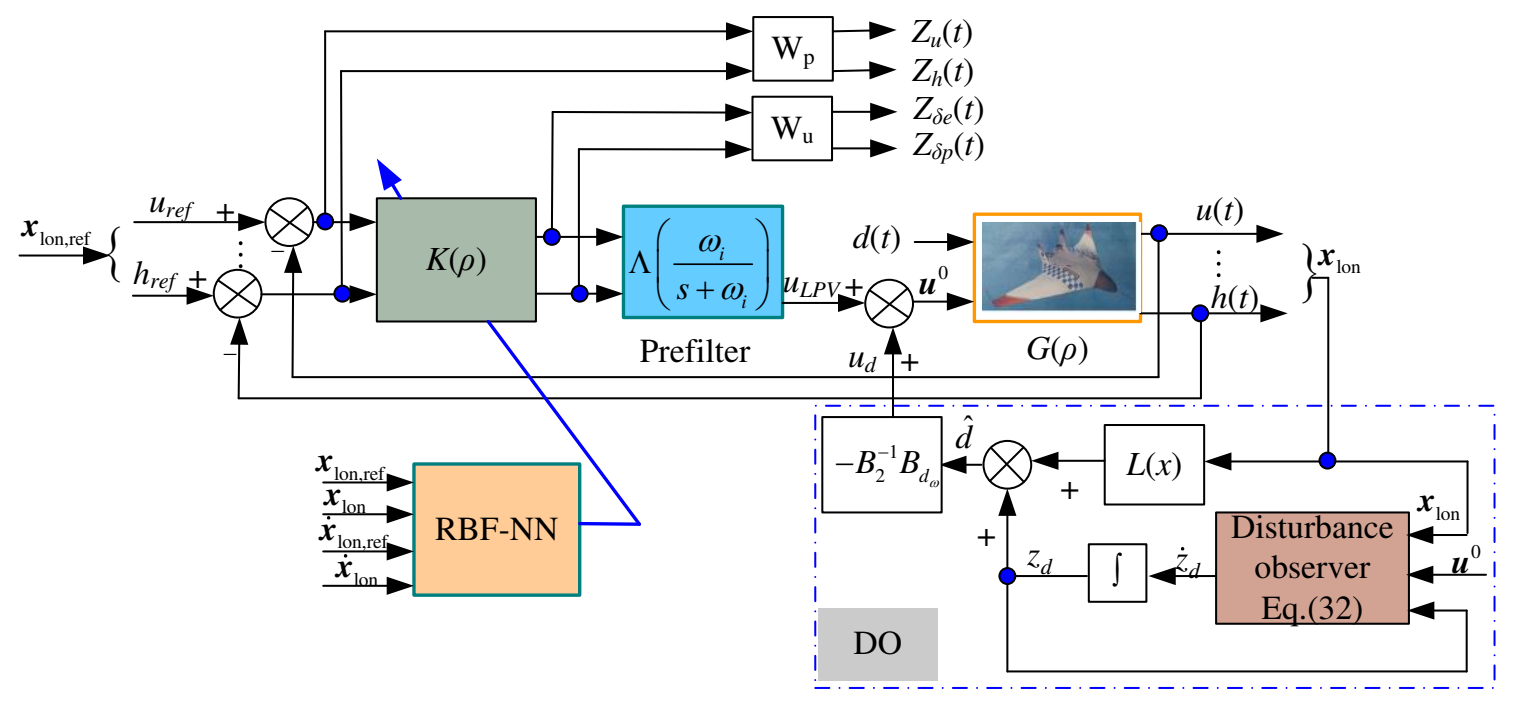

Fig. 3. Block diagram of of the DO-NNLPV controller for the BWB aircraft

According to above analysis, the observable disturbance $d(t)$ has been compensated by the DO based controller, and then the LPV controller can be designed by using the control synthesis based on SQLF in section III.A, hence, a proposition can be obtained as follows,

Proposition 1 There exists a composite controller of (37) (41) with a disturbance observer (32) that guarantees the closedloop system, (2) and (4), meets desired performances of $(a)$ and $(b)$, if the observer gain $L(x)$ is appropriately determined such that $W_{d}-L(x) B_{3} V_{d}$ is Hurwitz, $\gamma_{\mathrm{w}}$ of the RBFNN meets (114) (in Appendix), and the LMIs (29) (31) hold for some positive definite symmetric matrices $(X, Y)$, which further satisfy $\operatorname{rank}\left(X-Y^{-1}\right) \leq p$. 
Besides unknown disturbances acting on the BWB aircraft in-flight, there exist model uncertainties $\delta$ in the flight control system due to model mismatch of the original nonlinear model and LPV model. To handle with these uncertainties, the previous LPV Robust Control (LPVRC) and disturbance observer can be applied. In this paper a new Affine LPV Control (ALPVC) is proposed to stabilize the model uncertainties. The model mismatch uncertainties can be obtained by using the parameters of stability and control derivatives, thus the model is called an uncertain affine LPV model which is less conservative, for example, consider control derivative $M_{\delta e}$, which is related to aerodynamic coefficient $C_{m \delta}$, define $M_{\delta e_{\text {affine }}}$ to be depended affinely on $\rho$ (i.e., $u, h)$ as

$$
M_{\delta e_{\text {affine }}}=M_{\delta e_{0}}+u M_{\delta e_{u}}+h M_{\delta e_{h}}
$$

where $M_{\delta e_{0}}, M_{\delta e_{u}}, M_{\delta e_{h}}$ can be obtained by using a least-squares regression method [28]. Then mismatch uncertainty between a nonlinear $M_{\delta e}$ and an affine $M_{\delta e_{\text {affine }}}$ can be achieved as

$$
\tilde{\delta}_{M_{\delta e}}=M_{\delta e}-M_{\delta e_{\text {affine }}}
$$

$\tilde{\delta}_{M_{\delta e}}$ can be normalized as

$$
\delta_{M_{\delta e}}=\frac{\tilde{\delta}_{M_{\delta e}}-T_{M e}}{S_{M e}}, \delta_{M_{\delta e}} \in[-1,1] \text {, }
$$

where

$$
T_{M e}=\frac{\bar{\delta}_{M_{\delta e}}+\underline{\delta}_{M_{\delta e}}}{2}, S_{M e}=\frac{\bar{\delta}_{M_{\delta e}}-\underline{\delta}_{M_{\delta e}}}{2},
$$

Hence, the model (2) with consideration of model uncertainty can be transformed into the uncertain affine LPV model as follows [29]

$$
\left\{\begin{array}{l}
\dot{x}(t)=A(\rho, \delta) x(t)+B_{1}(\rho, \delta) \omega(t)+\left(B_{2} u(t)+B_{3} d(t)\right) \\
z(t)=C_{1}(\rho, \delta) x(t)+D_{11}(\rho, \delta) \omega(t)+D_{12} u(t) \\
y(t)=C_{2} x(t)+D_{21} \omega(t)
\end{array}\right.
$$

where $\delta=\left[\begin{array}{lll}\delta_{1} & \cdots & \delta_{m}\end{array}\right]^{T}$,

$$
\begin{gathered}
A(\boldsymbol{\rho}, \delta)=A(\boldsymbol{\rho})+\delta_{1} A_{\delta_{1}}(\boldsymbol{\rho})+\delta_{2} A_{\delta_{2}}(\boldsymbol{\rho})+\cdots+\delta_{m} A_{\delta_{m}}(\boldsymbol{\rho}), \\
A(\boldsymbol{\rho})=A(u, h)=\left(A_{0}+\sum_{i=1}^{m} T_{i} A_{\delta_{i, 0}}\right)+u\left(A_{u}+\sum_{i=1}^{m} T_{i} A_{\delta_{i, u}}\right)+h\left(A_{h}+\sum_{i=1}^{m} T_{i} A_{\delta_{i, h}}\right), \\
A_{\delta_{i}}(\boldsymbol{\rho})=A_{\delta_{i}}(u, h)=S_{i} A_{\delta_{i, 0}}+u S_{i} A_{\delta_{i, u}}+h S_{i} A_{\delta_{i, h}}, i=1,2, \ldots m .
\end{gathered}
$$


where $T_{i}$ and $S_{i}$ are the same as Eq.(45). By using the Linear Fraction Transform (LFT) technique [30], the parametric uncertainties $\delta$ in (46) can be separated from the system state-space model matrices as

$$
\begin{gathered}
{\left[\begin{array}{c}
\dot{x} \\
z_{\delta_{1}} \\
\vdots \\
z_{\delta_{m}} \\
z \\
y
\end{array}\right]=\left[\begin{array}{cccccc}
A(\cdot) & B_{\delta_{1}}(\cdot) \lambda^{-\frac{1}{2}} & \cdots & B_{\delta_{\delta_{m}}}(\cdot) \lambda^{-\frac{1}{2}} & B_{1}(\cdot) & B_{2} \\
\lambda^{\frac{1}{2}} C_{\delta_{\delta^{\prime}}}(\cdot) & \lambda^{\frac{1}{2}} D_{\delta \delta_{1}}(\cdot) \lambda^{-\frac{1}{2}} & \cdots & \lambda^{\frac{1}{2}} D_{\delta \delta_{\delta_{m}}}(\cdot) \lambda^{-\frac{1}{2}} & \lambda^{\frac{1}{2}} D_{\delta \delta_{1}}(\rho) & \lambda^{\frac{1}{2}} D_{\delta 2} \\
\vdots & & \vdots & & \\
\lambda^{\frac{1}{2}} C_{\delta_{m}}(\cdot) & \lambda^{\frac{1}{2}} D_{\delta \delta_{m_{1}}}(\cdot) \lambda^{-\frac{1}{2}} & & \lambda^{\frac{1}{2}} D_{\delta \delta_{m m}}(\cdot) \lambda^{-\frac{1}{2}} & \lambda^{\frac{1}{2}} D_{\delta_{1_{m}}}(\cdot) & \lambda^{\frac{1}{2}} D_{\delta 2} \\
C_{1}(\cdot) & D_{1 \delta_{1}}(\cdot) \lambda^{-\frac{1}{2}} & \cdots & D_{1 \delta_{m}}(\cdot) \lambda^{-\frac{1}{2}} & D_{11}(\cdot) & D_{12} \\
C_{2} & D_{2 \delta} \lambda^{-\frac{1}{2}} & \cdots & D_{2 \delta} \lambda^{-\frac{1}{2}} & D_{21} & 0
\end{array}\right]\left[\begin{array}{c}
x \\
\omega_{\delta_{1}} \\
\vdots \\
\omega_{\delta_{\delta_{m}}} \\
\omega \\
u
\end{array}\right]} \\
{\left[\begin{array}{c}
\omega_{\delta_{1}} \\
\omega_{\delta_{2}} \\
\vdots \\
\omega_{\delta_{m}}
\end{array}\right]=\left[\begin{array}{cccc}
\delta_{1} I_{S_{1}} & 0 & \cdots & 0 \\
0 & \delta_{2} I_{s_{2}} & \cdots & 0 \\
\vdots & & \ddots & \\
0 & 0 & \cdots & \delta_{m} I_{s_{m}}
\end{array}\right]\left[\begin{array}{c}
z_{\delta_{1}} \\
z_{\delta_{2}} \\
\vdots \\
z_{\delta_{m}}
\end{array}\right]}
\end{gathered}
$$

where $z_{\delta_{i}}, \omega_{\delta_{i}} \in \square^{S_{i}}, \lambda \in \lambda_{\Im}$ is a scaling matrix to be determined,

$$
\begin{gathered}
B_{\delta_{i}}(\rho)=B_{\delta_{i}}(u, h)=B_{\delta_{i, 0}}+u B_{\delta_{i, u}}+h B_{\delta_{i, h}} \\
C_{\delta_{i}}(\rho)=C_{\delta_{i}}(u, h)=C_{\delta_{i, 0}}+u C_{\delta_{i, u}}+h C_{\delta_{i, h}} \\
D_{\delta_{i i}}(\rho)=D_{\delta_{i i}}(u, h)=D_{\delta_{i i, 0}}+u D_{\delta_{i i, u}}+h D_{\delta_{i, i, h}}
\end{gathered}
$$

Denote

$$
\begin{gathered}
\omega_{\delta}=\left[\begin{array}{llll}
\omega_{\delta_{1}}^{T} & \omega_{\delta_{2}}^{T} & \cdots & \omega_{\delta_{m}}^{T}
\end{array}\right]^{T}, z_{\delta}=\left[\begin{array}{llll}
z_{\delta_{1}}^{T} & z_{\delta_{2}}^{T} & \cdots & z_{\delta_{m}}^{T}
\end{array}\right]^{T}, B_{\delta}(\rho)=\left[\begin{array}{llll}
B_{\delta_{1}}(\rho) & B_{\delta_{2}}(\rho) & \cdots & B_{\delta_{m}}(\rho)
\end{array}\right] \\
C_{\delta}(\boldsymbol{\rho})=\left[\begin{array}{llll}
C_{\delta_{1}}^{T}(\rho) & C_{\delta_{2}}^{T}(\rho) & \cdots & C_{\delta_{m}}^{T}(\rho)
\end{array}\right]^{T} \\
D_{\delta}(\boldsymbol{\rho})=\left[\begin{array}{cccc}
D_{\delta_{11}}(\rho) & D_{\delta_{12}}(\rho) & \cdots & D_{\delta_{1 m}}(\rho) \\
D_{\delta_{21}}(\rho) & D_{\delta_{22}}(\rho) & \cdots & D_{\delta_{2 m}}(\rho) \\
\vdots & & & \vdots \\
D_{\delta_{m 1}}(\rho) & D_{\delta_{m 2}}(\rho) & \cdots & D_{\delta_{m n}}(\rho)
\end{array}\right]
\end{gathered}
$$

Substituting (52) (57) into (50) (51) and yields

$$
\left[\begin{array}{c}
\dot{x} \\
z_{\delta} \\
z \\
y
\end{array}\right]=\left[\begin{array}{cccc}
A(\rho) & B_{\delta}(\rho) \lambda^{-\frac{1}{2}} & B_{1}(\rho) & B_{2} \\
\lambda^{\frac{1}{2}} C_{\delta}(\rho) & \lambda^{\frac{1}{2}} D_{\delta \delta}(\rho) \lambda^{-\frac{1}{2}} & \lambda^{\frac{1}{2}} D_{\delta 1_{1}}(\rho) & \lambda^{\frac{1}{2}} D_{\delta 2} \\
C_{1}(\rho) & D_{1 \delta}(\rho) \lambda^{-\frac{1}{2}} & D_{11}(\rho) & D_{12} \\
C_{2} & D_{2 \delta} \lambda^{-\frac{1}{2}} & D_{21} & 0
\end{array}\right]\left[\begin{array}{c}
x \\
\omega_{\delta} \\
\omega \\
u
\end{array}\right]
$$


where $z_{\delta}, \omega_{\delta} \in \square^{s}, s=s_{1}+s_{2}+\cdots+s_{m}$,

$$
\tilde{\Delta}=\operatorname{diag}\left(\delta_{1} I_{s_{1}}, \delta_{2} I_{s_{2}}, \delta_{m} I_{s_{m}}\right)
$$

Similarly, matrices of $B_{\delta}(\rho), B_{1}(\rho), C_{\delta}(\rho), C_{1}(\rho), D_{\delta \delta}(\rho), D_{\delta 1}(\rho), D_{1 \delta}(\rho)$, and $D_{11}(\rho)$ can be written as a convex combination of the matrix vertices as

$$
\left(\begin{array}{cccc}
A(\boldsymbol{\rho}) & B_{\delta}(\boldsymbol{\rho}) & B_{1}(\boldsymbol{\rho}) & B_{2} \\
C_{\delta}(\boldsymbol{\rho}) & D_{\delta \delta}(\boldsymbol{\rho}) & D_{\delta 1}(\boldsymbol{\rho}) & D_{\delta 2} \\
C_{1}(\boldsymbol{\rho}) & D_{1 \delta}(\boldsymbol{\rho}) & D_{11}(\boldsymbol{\rho}) & D_{12} \\
C_{2} & D_{2 \delta} & D_{21} & 0
\end{array}\right)=\sum_{i=1}^{r} \alpha_{i}\left(\begin{array}{cccc}
\hat{A}_{i} & \hat{B}_{\delta_{i}} & \hat{B}_{1_{i}} & B_{2} \\
\hat{C}_{\delta_{i}} & \hat{D}_{\delta \delta_{i}} & \hat{D}_{\delta 1_{i}} & D_{\delta 2} \\
\hat{C}_{1_{i}} & \hat{D}_{1 \delta_{i}} & \hat{D}_{11_{i}} & D_{12} \\
C_{2} & D_{2 \delta} & D_{21} & 0
\end{array}\right)
$$

By using the dynamic LPV controller, $K(\rho)$ as Eqs.(4) and (5), the closed-loop system of (4), (58) and (59) can be rewritten as (59) and

$$
\left[\begin{array}{c}
\dot{x}_{a u g} \\
z_{\delta} \\
z
\end{array}\right]=\left[\begin{array}{ccc}
A_{c l}(\boldsymbol{\rho}) & B_{\delta_{c l}}(\boldsymbol{\rho}) \lambda^{-\frac{1}{2}} & B_{1_{c l}}(\boldsymbol{\rho}) \\
\lambda^{\frac{1}{2}} C_{\delta_{c l}}(\boldsymbol{\rho}) & \lambda^{\frac{1}{2}} D_{\delta \delta_{c l}}(\boldsymbol{\rho}) \lambda^{-\frac{1}{2}} & \lambda^{\frac{1}{2}} D_{\delta 1_{c l}}(\boldsymbol{\rho}) \\
C_{1_{c l}}(\boldsymbol{\rho}) & D_{1 \delta_{c l}}(\boldsymbol{\rho}) \lambda^{-\frac{1}{2}} & D_{11_{c l}}(\boldsymbol{\rho})
\end{array}\right]\left[\begin{array}{c}
x_{a u g} \\
\omega_{\delta} \\
\omega
\end{array}\right]
$$

where $x_{\text {aug }}=\left[\begin{array}{c}x(t) \\ x_{k}(t)\end{array}\right]$,

$$
\begin{aligned}
& B_{\delta_{c l}}(\boldsymbol{\rho})=\left[\begin{array}{c}
B_{\delta}(\boldsymbol{\rho}) \\
0
\end{array}\right]+\bar{B} K(\boldsymbol{\rho}) \bar{D}_{\delta_{21}}=\sum_{i=1}^{r} \alpha_{i} \hat{B}_{\delta_{c_{i}}}, \\
& \hat{B}_{\delta_{c_{i}}}=\left[\begin{array}{c}
\hat{B}_{\delta_{i}} \\
0
\end{array}\right]+\bar{B} K_{i} \bar{D}_{\delta_{21}}, \\
& C_{\delta_{c l}}(\rho)=\left[\begin{array}{ll}
C_{\delta}(\rho) & 0
\end{array}\right]+\bar{D}_{\delta_{12}} K(\rho) \bar{C}=\sum_{i=1}^{r} \alpha_{i} \hat{C}_{\delta_{c i}}, \\
& \hat{C}_{\delta_{c_{i}}}=\left[\begin{array}{ll}
\hat{C}_{\delta_{i}} & 0
\end{array}\right]+\bar{D}_{1_{12}} K_{i} \bar{C}, \\
& D_{\delta \delta_{c l}}(\rho)=D_{\delta \delta}+\bar{D}_{\delta_{12}} K(\rho) \bar{D}_{\delta_{21}}=\sum_{i=1}^{r} \alpha_{i} \hat{D}_{\delta \delta_{c_{i}}}, \\
& \hat{D}_{\delta \delta_{c_{i}}}=D_{\delta \delta_{i}}+\bar{D}_{\delta_{12}} K_{i} \bar{D}_{\delta_{21}}, \\
& D_{\delta 1_{c l}}(\boldsymbol{\rho})=D_{\delta 1}+\bar{D}_{\delta_{12}} K(\boldsymbol{\rho}) \bar{D}_{\mathrm{l}_{21}}=\sum_{i=1}^{r} \alpha_{i} \hat{D}_{\delta 1_{c_{i}}}, \\
& \hat{D}_{\delta 1_{c_{i}}}=D_{\delta 1_{i}}+\bar{D}_{\delta_{12}} K_{i} \bar{D}_{1_{21}},
\end{aligned}
$$




$$
\begin{gathered}
D_{1 \delta_{c l}}(\rho)=D_{1 \delta}+\bar{D}_{1_{12}} K(\rho) \bar{D}_{\delta_{21}}=\sum_{i=1}^{r} \alpha_{i} \hat{D}_{1 \delta_{c i}}, \\
\hat{D}_{1 \delta_{c c_{i}}}=D_{1 \delta_{i}}+\bar{D}_{1_{12}} K_{i} \bar{D}_{\delta_{21}}, \\
D_{\delta_{21}}=\left[\begin{array}{c}
0 \\
D_{2 \delta}
\end{array}\right], D_{\delta_{12}}=\left[\begin{array}{ll}
0 & D_{\delta 2}
\end{array}\right], \bar{D}_{1_{12}}=\left[\begin{array}{ll}
0 & D_{12}
\end{array}\right],
\end{gathered}
$$

the other matrix parameters are the same as in (7)-(11). By Proposition 1, we have following conclusion,

Theorem 1 There exists a composite controller of (37) (41) with a disturbance observer (32) that guarantees the closed-loop system, (46) and (4), meet desired performances of $(a)$ and $(b)$, if the observer gain $L(x)$ is appropriately determined such that $W_{d}-L(x) B_{3} V_{d}$ is Hurwitz, $\gamma_{\mathrm{w}}$ of the RBFNN meets (114) (in Appendix), and the following LMI conditions hold for some positive definite symmetric matrices $(R, S)$, which further satisfy $\operatorname{rank}\left(R-S^{-1}\right) \leq p$.

$$
\begin{gathered}
\left(\begin{array}{cc}
N_{R} & 0 \\
0 & I
\end{array}\right)^{T}\left[\begin{array}{ccccc}
\hat{A}_{i}^{T} R+R \hat{A}_{i} & R \hat{B}_{\delta_{i}} & R \hat{B}_{1_{i}} & \hat{C}_{\delta_{i}}^{T} & \hat{C}_{1_{i}}^{T} \\
* & -\gamma \lambda & 0 & \hat{D}_{\delta \delta_{i}}^{T} & \hat{D}_{1 \delta_{i}}^{T} \\
* & * & -\gamma I & \hat{D}_{\delta 1_{i}}^{T} & \hat{D}_{11_{i}}^{T} \\
* & * & * & -\gamma \lambda & 0 \\
* & * & * & * & -\gamma I
\end{array}\right]\left(\begin{array}{cc}
N_{R} & 0 \\
0 & I
\end{array}\right)<0 \\
\left(\begin{array}{cc}
N_{S} & 0 \\
0 & I
\end{array}\right)^{T}\left[\begin{array}{ccccc}
\hat{A}_{i} S+S \hat{A}_{i}^{T} & S \hat{C}_{\delta_{i}}^{T} & S \hat{C}_{1_{i}}^{T} & \hat{B}_{\delta_{i}} & \hat{B}_{1_{i}} \\
* & -\gamma \lambda & 0 & \hat{D}_{\delta \delta_{i}}^{T} & \hat{D}_{\delta 1_{i}}^{T} \\
* & * & -\gamma I & \hat{D}_{1 \delta_{i}}^{T} & \hat{D}_{11_{i}}^{T} \\
* & * & * & -\gamma \lambda & 0 \\
* & * & * & * & -\gamma I
\end{array}\right]\left(\begin{array}{cc}
N_{S} & 0 \\
0 & I
\end{array}\right)<0 \\
\\
\left(\begin{array}{cc}
R & I \\
I & S
\end{array}\right)>0
\end{gathered}
$$

for $\mathrm{i}=1, \ldots, \mathrm{r}$, where* denotes a symmetric matrix element.

Proof. See the Appendix.

Remark 2 Although (74)-(76) are not standard LMI problems due to the scale matrix $\lambda$, they can be solved by an iterative approach, referred to as D-K iteration [25]. Like the $\mu$-synthesis algorithms, such a scheme is not guaranteed to converge to a global minimum, but may find a local minimum [25]. In spite of this drawback, the D-K iteration control design technique appears to work well on many engineering problems. 
Remark 3 For the problem of trajectory tracking control, the Lyapunov function $V_{c}=x^{T} P x$ can be modified as $V_{c}=e^{T} P e$, thus the closed-loop error system is guaranteed to be stable, hence the input vector of the RBF neural network are chosen as $x_{N N}=\left[\begin{array}{ll}e^{T} & \dot{e}^{T}\end{array}\right]^{T}$.

Now we return to design the trajectory tracking control as in Fig.3. The objective of mixed-sensitivity loop-shaping is to shape the sensitivity function $S$ and control sensitivity function $K \cdot S$ with performance weighting functions $W_{\mathrm{p}}$ and robustness weighting functions $W_{\mathrm{u}}$, respectively. The desired controller meets the requirements of small tracking error, attenuation of the effect of disturbance on output, low sensitivity to measurement noise, with reasonably small control effort, and is robustly stable to additive plant perturbations. Hence, the mixed-sensitivity criterion is employed as follows,

$$
\| \begin{gathered}
W_{p} S \\
W_{u} K S \|_{\infty}<1
\end{gathered}
$$

To have desired tracking performances and robustness, the performance weighting function $W_{\mathrm{p}}$, is selected as

$$
\begin{gathered}
W_{p_{u}}=\frac{0.5 s+0.65}{s+0.001 \times 0.65}, W_{p_{h}}=\frac{0.5 s+0.65}{s+0.001 \times 0.65}, \quad \text { (without model uncertainties) } \\
W_{p_{u}}=\frac{0.5 s+2.64}{s+0.001 \times 2.64}, W_{p_{h}}=\frac{0.5 s+0.6}{s+0.001 \times 0.6}, \quad \text { (with model uncertainties) }
\end{gathered}
$$

The robustness weighting function $W_{\mathrm{u}}$ is selected as follows

$$
\begin{gathered}
W_{u_{\delta_{p}}}=\frac{s+0.65 / 2}{0.001 s+0.65}, W_{u_{\delta_{e}}}=\frac{s+0.65 / 2}{0.001 s+0.65}, \quad \text { (without model uncertainties) } \\
W_{u_{\delta_{p}}}=\frac{0.01 \times(s+26.4 / 2)}{0.001 s+26.4}, W_{u_{\delta_{e}}}=\frac{100 \times(s+6 / 2)}{0.001 s+6}, \quad \text { (with model uncertainties) }
\end{gathered}
$$

The purpose of $W_{\text {prefilter }}$ is to make matrices $B_{2}$ and $D_{12}$ of the plant model to be parameter-independent, hence, according to the gain-scheduled dynamic feedback $H_{\infty}$ controller design [16], the transfer function $W_{\text {prefilter }}$ is chosen as

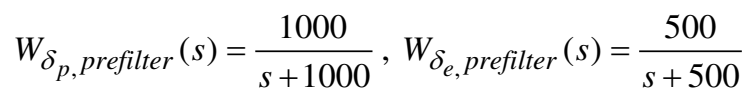

Once the longitudinal affine LPV model given by Eq.(16) and (61) are augmented with the weighting functions shown in (78) (82), then a pair of positive definite symmetric matrices $(R, S)$ can be determined using Proposition 1 and Theorem 1 , for which the performance measure $(\gamma)$ can be obtained. 


\section{Numerical Simulation and Analysis}

To illustrate the DO-NNSQLF based LPV design, first an LPV model of the BWB aircraft designed by Cranfield University is constructed. The BWB aircraft flight at $0 \mathrm{~m}$ altitude and $191.9 \mathrm{~m} / \mathrm{s}$ speed, the trim state

$$
\begin{aligned}
& x_{e}=\left[\begin{array}{llllllllllll}
u & w & q & \theta & h & x & v & p & r & \phi & \psi & y
\end{array}\right]_{e}^{T}
\end{aligned}
$$

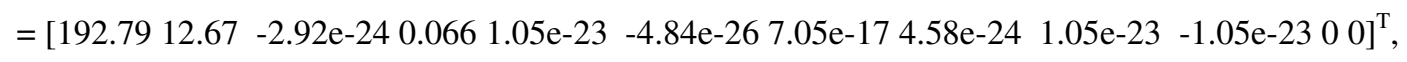

unit: [u v w] (m/s), [p q r] (rad/s), [x y h] (m), [ $\phi, \theta, \psi](\mathrm{rad}) . u_{e}=\left[\begin{array}{llll}\delta_{e} & \delta_{p} & \delta_{a} & \delta_{r}\end{array}\right]_{e}^{T}=[-0.110 .28-6 \mathrm{e}-19-1.71 \mathrm{e}-19]^{\mathrm{T}}$, unit:

$\left[\delta_{\mathrm{a}} \delta_{\mathrm{e}} \delta_{\mathrm{r}}\right](\mathrm{rad})$

The position range for the actuators are $\delta_{\mathrm{e}} \in[-\pi / 6, \pi / 6](\mathrm{rad}), \delta_{\mathrm{p}} \in[0,1], \delta_{\mathrm{a}} \in[-\pi / 6, \pi / 6](\mathrm{rad}), \delta_{\mathrm{r}} \in[-\pi / 6, \pi / 6](\mathrm{rad})$. The initial position $\xi_{0}=[0,0,0]^{\mathrm{T}}(\mathrm{m})$, and initial body velocity $\boldsymbol{V}_{0}=[192 \mathrm{~m} / \mathrm{s}, 0,0]^{\mathrm{T}}$, initial attitude $\eta_{0}=\left[0,5.5^{\circ}, 0\right]^{\mathrm{T}}$, initial angular velocity $\omega_{0}=[0,0,0]^{\mathrm{T}}$, and flight envelope is $(u, h) \in([167,218]) \times([0,3048])(\mathrm{m} / \mathrm{s}, \mathrm{m})$.

Scenario I: Trajectory tracking control under unknown disturbances.

In this case the LPV model of longitudinal motion is as

$$
\dot{x}(t)=A(\rho) x(t)+B(\rho) u(t)
$$

where state $x=\left[\begin{array}{lllll}u & \omega & q & \theta & h\end{array}\right]^{T}$, and control $u_{\mathrm{lon}}=\left[\begin{array}{ll}\delta_{e} & \delta_{\mathrm{p}}\end{array}\right]^{T}$,

$$
\begin{aligned}
& A(\rho)=A(u, h)=A_{0}+u A_{u}+h A_{h}, B(\rho)=B(u, h)=B_{0}+u B_{u}+h B_{h} \\
& A_{0}=\left[\begin{array}{cccccc}
0.0115 & -0.1524 & -26.9900 & -9.4307 & 0 \\
-0.0715 & -0.3968 & -0.8695 & -3.0135 & 0 \\
0.0053 & -0.0112 & -0.1293 & 0 & 0 \\
0 & 0 & 1.0000 & 0 & 0 \\
0.3076 & -0.9616 & 0 & 3.9702 & 0
\end{array}\right], A_{u}=\left[\begin{array}{ccccc}
-0.0001 & 0.0005 & 0.0786 & -0.0018 & 0 \\
0.0003 & -0.0045 & 0.9886 & 0.0012 & 0 \\
-0.0000 & -0.0002 & -0.0017 & 0 & 0 \\
0 & 0 & 0 & 0 & 0 \\
-0.0012 & -0.0002 & 0 & 0.9806 & 0
\end{array}\right]
\end{aligned}
$$

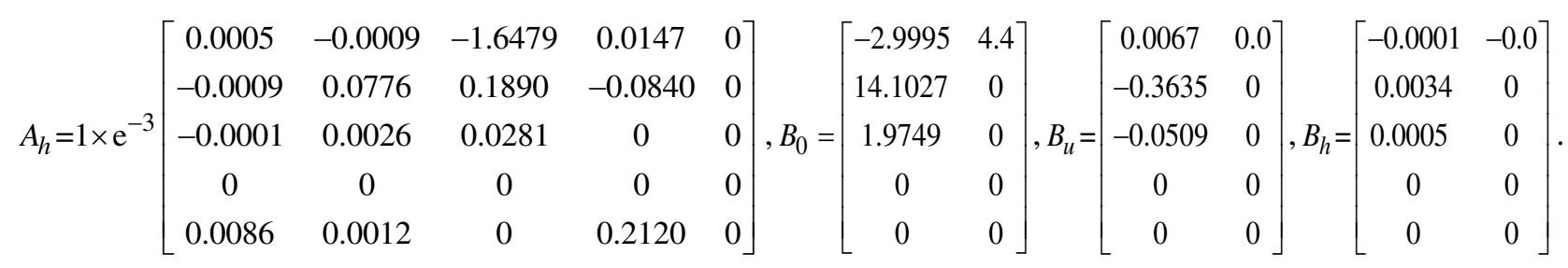

In this scenario suppose that there is the unknown disturbance vector $d$ acting on the BWB aircraft, given by

$$
d(t)=\left[\begin{array}{ll}
d_{\delta_{e}} & d_{\delta_{p}}
\end{array}\right]^{T}=\left[\begin{array}{ll}
0.05 \sin (0.05 t)(\operatorname{rad}) & 0.1 \cos (0.05 t)
\end{array}\right]^{T}
$$

The weight matrices of $W_{d}$ and $V_{d}$ are as 


$$
W_{d}=\left[\begin{array}{cc}
0 & 0.05 \\
-0.05 & 0
\end{array}\right], V_{d}=\left[\begin{array}{cc}
3.5 & 0 \\
0 & 7
\end{array}\right]
$$

The gains of the disturbance observer (32) are designed as follows according to section III.B and Remark 1 ,

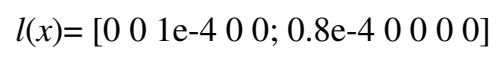

By Proposition 1, the proposed control parameter $K_{\mathrm{i}}(i=1,2,3,4)$ are obtained, and then by using (4) the dynamic controller is achieved, and a PDLF controller ${ }^{[31]}$ is used to compare with the proposed SQLF controller. the performance obtained through the DO-SQLF and DO-PDLF approaches are $\gamma=1.3689$. By using the proposed controller, the trajectory tracking responses are shown in Figs 4-7,

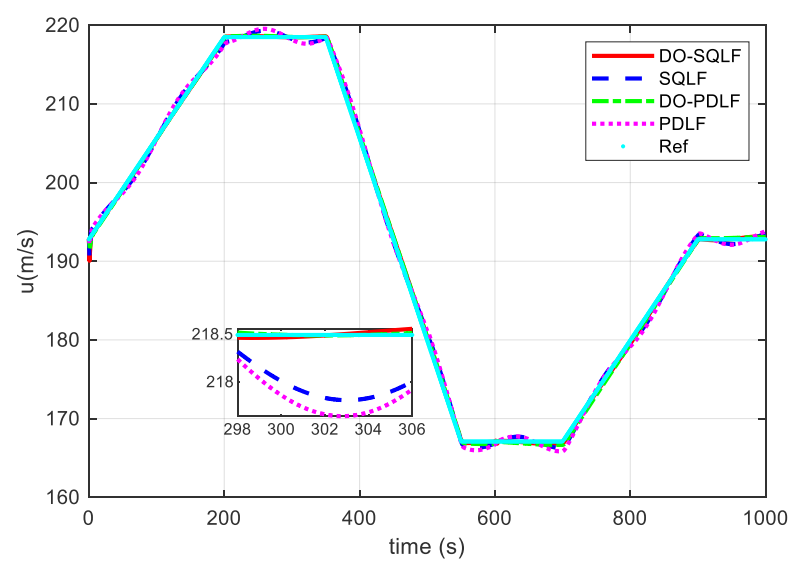

Fig. 4. Forward speed tracking output under unknown disturbances.

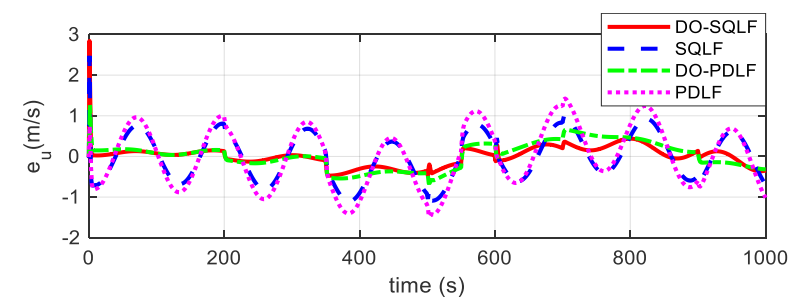

Fig.5. Tracking error response of forward speed

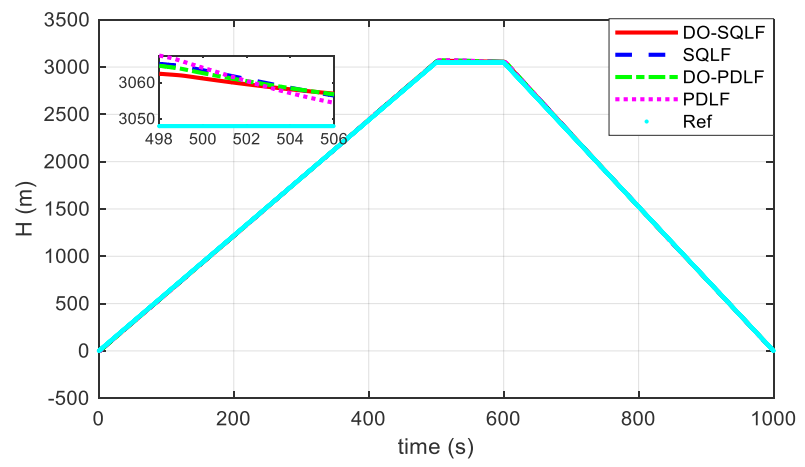

Fig.6 Altitude tracking output under unknown disturbances 


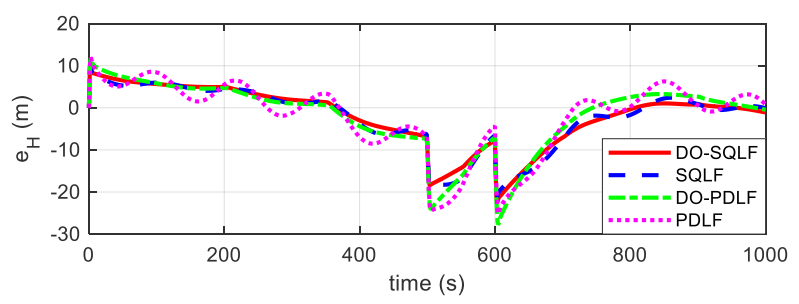

Fig.7 Tracking error response of altitude

From Figs 4-7, it can be seen that the desired forward velocity and altitude can be tracked precisely by the proposed DO-SQLF LPV control. Comparing with PDLF design, responses of the SQLF control have less tracking errors due to its more dependence on precisely model for PDLF although it can reduce control conservative. Meanwhile, comparing with SQLF design without DO, there are less steady tracking errors than for the SQLF LPV design, this shows DO-SQLF design can compensate for observable disturbances and make their responses smooth and easily approach the desired trajectories, the same case is for PDLF design. NNSQLF has also been simulated, the responses are listed as Figs 8-9.

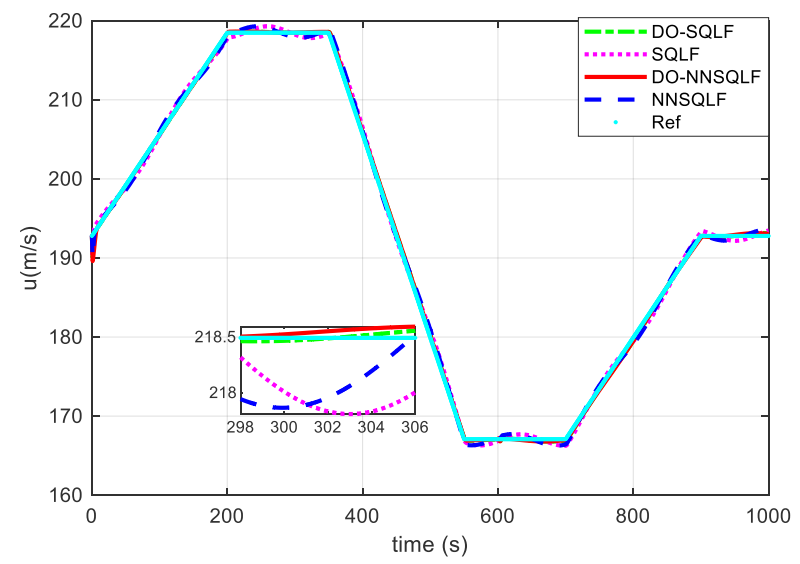

Fig.8 Forward speed tracking output under unknown disturbances

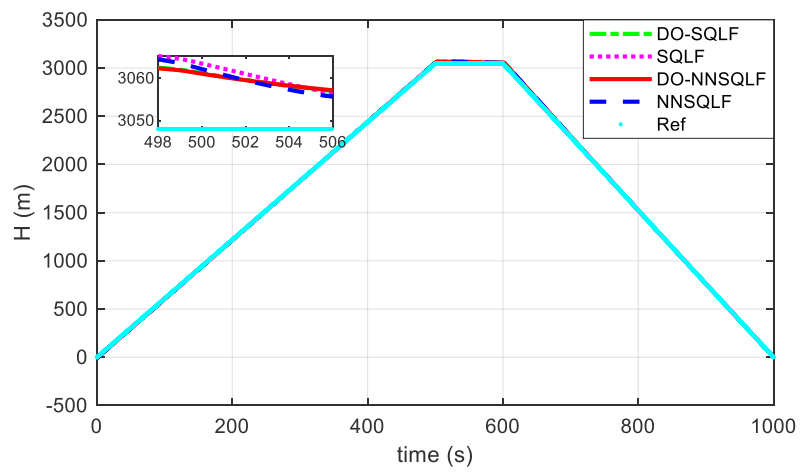

Fig.9 Altitude tracking output under unknown disturbances

The associated control inputs are shown in Fig.10. 


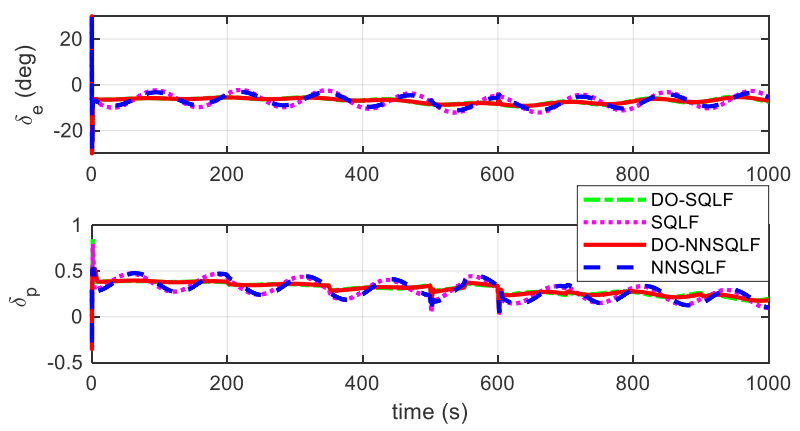

Fig.10 control inputs, in Scenario 1

Comparing with SQLF design, responses of the NNSQLF based LPV control have less overshoot and tracking errors, see Figs 8-9, which shows RBF has better model approximate capability when the plant exists model errors. From Fig. 10 it can be seen that the elevator and throttle inputs of the NNSQLF LPV control are greater than those of the DO-NNSQLF LPV control. The unknown observable disturbances have been estimated via DO as shown in Fig.11.

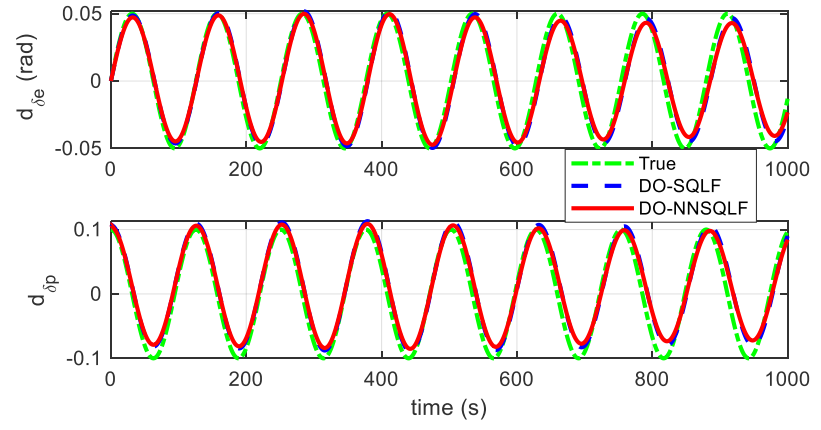

Fig.11 Disturbance estimations of elevator and thrust inputs

From Fig.11 it can be seen that estimation values of the actuator disturbances approach the true ones, and their estimation errors are small within the admissible range. The harmonic wave disturbance frequency of the true values and their estimation ones are same.

\section{Scenario II: Trajectory tracking control under model uncertainty with unknown disturbances.}

In this scenario the LPV model of longitudinal motion is as

$$
\dot{x}(t)=A(u, h, \delta) x(t)+B(u, h, \delta) u(t)
$$

where

$$
A(u, h, \delta)=A_{0}+A_{e p}+u \tilde{A}_{u}+h \tilde{A}_{h}, \quad B(u, h, \delta)=B_{0}+B_{e p}+u \tilde{B}_{u}+h \tilde{B}_{h}
$$


$A_{0}=\left[\begin{array}{ccccc}0.0063778 & -0.13032 & -20.555 & -9.701 & 0 \\ -0.065984 & -0.19224 & -0.42574 & -1.7615 & 0 \\ 0.004467 & -0.0054977 & -0.063313 & 0 & 0 \\ 0 & 0 & 1 & 0 & 0 \\ 0.1796 & -0.98922 & 0 & 1.5195 & 0\end{array}\right], \tilde{A}_{h}=\left[\begin{array}{ccccc}1.7763 \mathrm{e}-7 & -6.3938 \mathrm{e}-07 & -0.000307 & 2.1215 \mathrm{e}-6 & 0 \\ -5.3202 \mathrm{e}-7 & 3.3096 \mathrm{e}-5 & 8.037 \mathrm{e}-5 & -1.5524 \mathrm{e}-5 & 0 \\ -1.9158 \mathrm{e}-8 & 1.0995 \mathrm{e}-6 & 1.1952 \mathrm{e}-5 & 0 & 0 \\ 0 & 0 & 0 & 0 \\ 1.5909 \mathrm{e}-6 & 1.2083 \mathrm{e}-7 & 0 & 2.2904 \mathrm{e}-5 & 0\end{array}\right]$

$A_{e p}=\left[\begin{array}{ccccc}2.0883 \mathrm{e}-5 \delta_{X u} & -0.00027913 \delta_{X w} & -0.15559 \delta_{X q} & 0.0025215 \delta_{X \theta} & 0 \\ -0.00016 \delta_{Z u} & -0.0063239 \delta_{Z w} & -0.014673 \delta_{Z q} & -0.017425 \delta_{Z \theta} & 0 \\ 1.0807 \mathrm{e}-05 \delta_{M u} & -0.0001959 \delta_{M w} & -0.0021821 \delta_{M q} & 0 & 0 \\ 0 & 0 & 0 & 0 & 0 \\ 0.0017828 \delta_{h u} & 0.00025771 \delta_{h w} & 0 & 0.03275 \delta_{h \theta} & 0\end{array}\right], \tilde{A}_{u}=\left[\begin{array}{ccccc}-2.653 \mathrm{e}-05 & 0.00023958 & 0.020817 & -0.00022431 & 0 \\ 0.00014241 & -0.0030938 & 0.50653 & 0.0029681 & 0 \\ -4.0526 \mathrm{e}-06 & -0.00010397 & -0.0011255 & 0 \\ 0 & 0 & 0 & 0 \\ -0.00030281 & -2.2885 \mathrm{e}-05 & 0 & 0.51116 & 0\end{array}\right]$

$B_{e p}=\left[\begin{array}{cc}0.018241 \delta_{X \delta_{e}} & -2.629 \mathrm{e}-13 \delta_{X} \delta_{p} \\ -0.88089 & 0 \\ -0.12336 & 0 \\ 0 & 0 \\ 0 & 0\end{array}\right], B_{0}=\left[\begin{array}{cc}-3.6318 & 4.4441 \\ 41.829 & 0 \\ 5.8577 & 0 \\ 0 & 0 \\ 0 & 0\end{array}\right], \tilde{B}_{u}=\left[\begin{array}{cc}0.0052267 & 1.2483 \mathrm{e}-14 \\ -0.26457 & 0 \\ -0.03705 & 0 \\ 0 & 0 \\ 0 & 0\end{array}\right]$,

$$
\tilde{B}_{h}=\left[\begin{array}{cc}
-2.7552 \mathrm{e}-5 & 5.1348 \mathrm{e}-17 \\
0.0014081 & 0 \\
0.0001972 & 0 \\
0 & 0 \\
0 & 0
\end{array}\right]
$$

$\delta_{i} \in[-1,1]$, the uncertainty in (88) can be separated as (50) and (51). The unknown disturbance vector $d$ acting on the BWB aircraft is the same as (85), the weight matrices of $W_{d}$ and $V_{d}$ are as

$$
W_{d}=\left[\begin{array}{cc}
0 & 0.05 \\
-0.05 & 0
\end{array}\right], V_{d}=\left[\begin{array}{cc}
30 & 0 \\
0 & 60
\end{array}\right] \text {, }
$$

the gains of the disturbance observer (32) are designed as

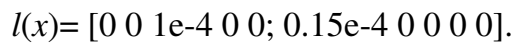

By Theorem 1, the proposed control parameter $K_{\mathrm{i}}(i=1,2,3,4)$ can be obtained, and then by using (4) the dynamic controller is achieved, the scale matrix

$\lambda=\operatorname{diag}(61433,61422,61261,61434,61412,54100,61427,61433,61461,61452,61461,60746,61431,61433,61433,61434,59454)$

for all case, for which the performance obtained is $\gamma=2.634$.

For the comparison analysis, the LPVRC for the model uncertainties $\delta$ is used here. For simplification, assume model uncertainties $\delta$ result the aerodynamic coefficients varying, that is, 


$$
\left[\begin{array}{cccccc}
\Delta C_{x} & \Delta C_{y} & \Delta C_{z} & \Delta C_{l} & \Delta C_{m} & \Delta C_{n}
\end{array}\right]^{T}=-(0.3 \alpha) \times\left[\begin{array}{llllll}
C_{x} & C_{y} & C_{z} & C_{l} & C_{m} & C_{n}
\end{array}\right]^{T},
$$

where $\alpha$ is angle of attack. By using the proposed DO-NNSQLF controller in the proposition 1 (NNLPVRC), and Theorem 1 $(N N A L P V C)$, the trajectory tracking responses are as Figs12-13,

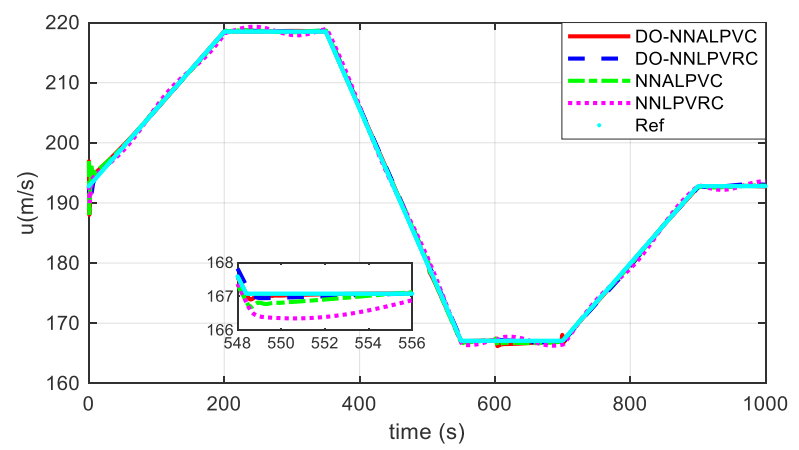

Fig.12 Forward speed tracking output under model uncertainties and unknown disturbances

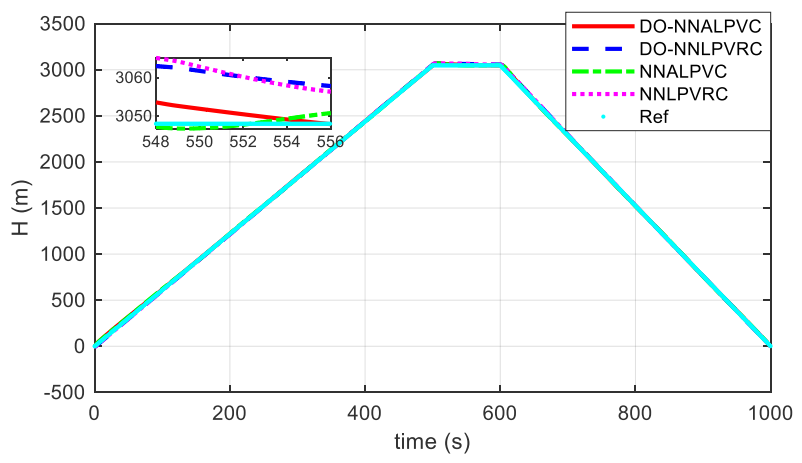

Fig.13 Altitude responses under model uncertainties and disturbances

From Figs 12 and 13, it can be seen that the command inputs of $\mathrm{u}_{\mathrm{ref}}$ and $\mathrm{h}_{\mathrm{ref}}$ are tracked well by the proposed DO-NNSQLF LPV controllers. In comparison with LPVRC control (regards model uncertainties as disturbances), NNALPVC has more precision and smaller overshoots of the responses. Meanwhile responses of the DO-NNSQLF LPV control have smaller tracking errors than those of the NNSQLF design without DO. This demonstrates the DO-NNSQLF LPV control can compensate for observable disturbances with smooth response and thus enhance the trajectory tracking control capability. The associated responses of the vertical speed, pitch motion are shown in Fig.14. 

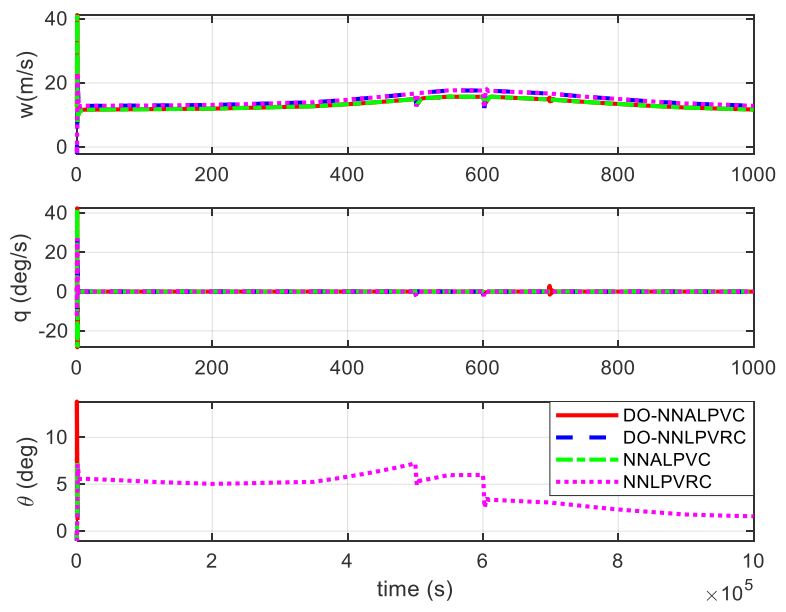

Fig.14 Responses of vertical speed, pitch angle and pitch rate

Fig.14 shows that there are larger fluctuations of the responses of the vertical speed, pitch angle and pitch rate by the NNSQLF design without DO than by the DO-NNSQLF control. This is because the DO-NNSQLF LPV control can reduce the unknown disturbance effect by disturbance-observer compensation. The control inputs are shown in Fig.15.
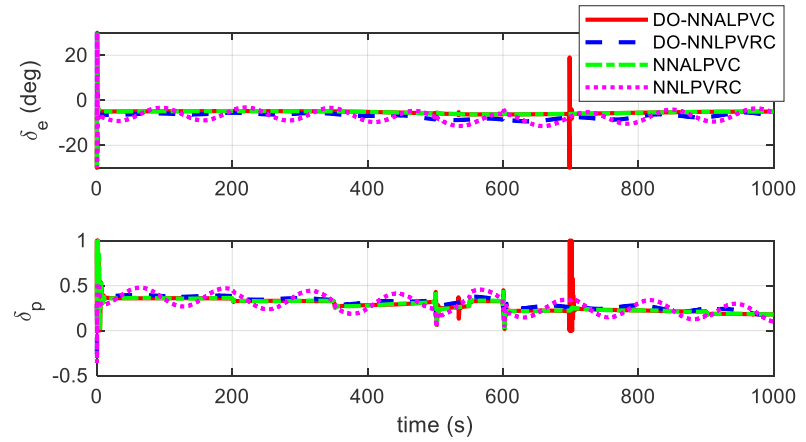

Fig.15 Control inputs of the elevator and thrust

Fig.15 shows that the NNSQLF LPV control with DO requires smaller control inputs than those of NNSQLF design without DO. The disturbances have been observed via DOs as Fig.16.

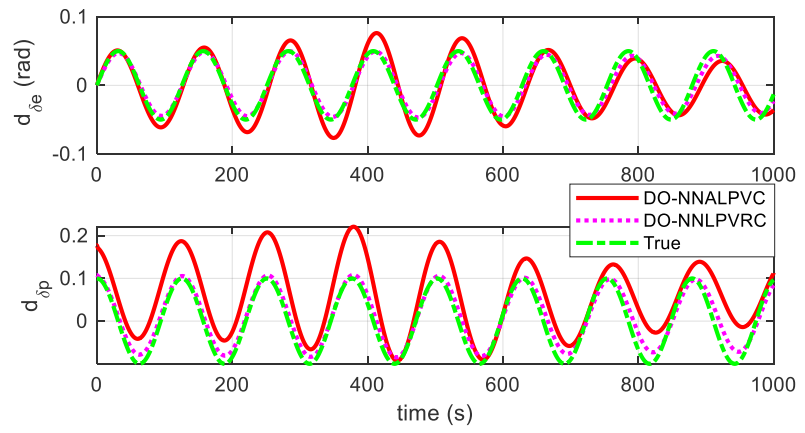

Fig.16 Disturbance estimations of elevator and thrust inputs

Fig.16 shows that disturbance estimations by NNLPVRC and NNALPVC are asymptotically convergent to the true values, but 
there exist some errors in transition stage because the disturbance estimations are varying with the associated states. Meanwhile it can be seen that it is more easily affected by the flight speed and altitude for NNALPVC tracking outputs than for the NNLPVRC ones, especially for thrust disturbance estimation, this is because the NNALPVC is sensible for flight parameters.

\section{CONCLUSION AND FUTURE WORK}

The problem of robust flight control for a BWB aircraft with model uncertainties and unknown disturbances has been addressed in this paper. A novel approach to dynamic tracking control of a BWB aircraft has been presented using the DONNSQLF based LPV design. The design of the system stabilization with model uncertainties and robust design for the requirement of the desired output responses under rejecting disturbances are investigated. Simulation results of altitude and speed trajectory tracking control of the BWB aircraft show that the trajectory tracking performances of the proposed controller are met desired requirements in the large flight envelope. Since machine learning can improve the LPV control performances, the future work is to enhance robustness and adaptive performance for the BWB aircraft by using that reinforcement learning and genetic algorithms.

\section{Appendix}

Proof. Based on the SQLF method, there is an LPV controller $K(\rho)$ that stabilizes the closed-loop system (46) and (4) and guarantees the quadratic $H_{\infty}$ performance of $(b)$ is bounded by $\gamma$ along all possible parameter trajectories, $\forall \boldsymbol{\rho} \in \Theta$, if and only if there exists $P=P^{\mathrm{T}}$ such that [31]

$$
P>0, \frac{d}{d t}\left(x^{T} P x\right)+z^{T} z+z_{\delta}^{T} z-\gamma^{2}\left(\omega^{T} \omega+\omega_{\delta}^{T} \omega_{\delta}\right)<0, \forall \rho \in \Theta,
$$

By using bounded real lemma [30], (94) leads the following scaled inequality

$$
\left[\begin{array}{ccccc}
A_{c l}^{T}(\rho) P+P A_{c l}(\rho) & P B_{\delta_{c l}}(\rho) & P B_{1_{c l}}(\rho) & C_{\delta_{c l}}^{T}(\rho) & C_{1_{c l}}^{T}(\rho) \\
* & -\gamma \lambda & 0 & D_{\delta \delta_{c l}}^{T}(\rho) & D_{1 \delta_{c l}}^{T}(\rho) \\
* & * & -\gamma I & D_{\delta 1_{c l}}^{T}(\rho) & D_{11_{c l}}^{T}(\rho) \\
* & * & * & -\gamma \lambda & 0 \\
* & * & * & * & -\gamma I
\end{array}\right]<0
$$

Substituting (61) into (95) and yields 


$$
\sum_{i=1}^{r} \alpha_{i}\left[\begin{array}{ccccc}
\hat{A}_{c l_{i}}^{T} P+P \hat{A}_{c l_{i}} & P \hat{B}_{\delta_{c l_{i}}} & P \hat{B}_{1_{c l_{i}}} & \hat{C}_{\delta_{c l_{i}}}^{T} & \hat{C}_{1_{c l_{i}}}^{T} \\
* & -\gamma \lambda & 0 & \hat{D}_{\delta \delta_{c l_{i}}}^{T} & \hat{D}_{1 \delta_{c l_{i}}}^{T} \\
* & * & -\gamma I & \hat{D}_{\delta 1_{c l_{i}}}^{T} & \hat{D}_{11_{c l_{i}}}^{T} \\
* & * & * & -\gamma \lambda & 0 \\
* & * & * & * & -\gamma I
\end{array}\right]<0,
$$

Inequality (96) can also be rewritten as [26]

$$
\sum_{i=1}^{r} \alpha_{i}\left(\Psi_{c l}+\bar{Q}^{T} K_{i}^{T} \bar{P}_{c l}+\bar{P}_{c l}^{T} K_{i} \bar{Q}\right)<0
$$

where $\Psi_{c l_{i}}=$

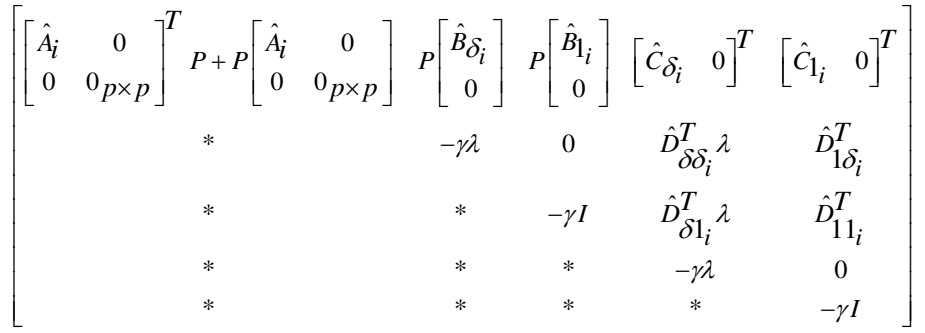

$$
\begin{aligned}
& \bar{Q}=\left[\begin{array}{llll}
\bar{C} & \bar{D}_{\delta_{21}} & \bar{D}_{1_{21}} & 0_{\left(p+q_{2}\right) \times q_{1}}
\end{array}\right], \bar{P}_{c l}=\left[\begin{array}{llll}
\bar{B}^{T} P & 0_{\left(p+m_{2}\right) \times m_{1}} & \bar{D}_{\delta_{12}}^{T} & \bar{D}_{1_{12}}^{T}
\end{array}\right],
\end{aligned}
$$

If the quadratic Lyapunov variable $P \in \square^{2 p \times 2 p}$ is obtained, the system matrix vertices $K_{i}$ of the LPV controller $K(\rho)$ for each vertex $i, i=1, \ldots, \mathrm{r}$, can be determined from (97). By Lemma 1, the LMIs (97) are sufficiently evaluated at all vertices. When $K_{i}$ is known, the controller system matrices $A_{\mathrm{k}}(\rho), B_{\mathrm{k}}(\rho), C_{\mathrm{k}}(\rho), D_{\mathrm{k}}(\rho)$ can be computed on-line in real-time using (4) with instantaneous measurement values of $\rho$. By Lemma 2, the LMIs (97) are solvable for $K_{i}$ if and only if there exist a pair of positive definite symmetric matrices $(R, S)$ satisfying

$$
\begin{gathered}
\sum_{i=1}^{r} \alpha_{i}\left(\begin{array}{cc}
N_{R} & 0 \\
0 & I
\end{array}\right)^{T}\left[\begin{array}{ccccc}
\hat{A}_{i}^{T} R+R \hat{A}_{i} & R \hat{B}_{\delta_{i}} & R \hat{B}_{1_{i}} & \hat{C}_{\delta_{i}}^{T} & \hat{C}_{1_{i}}^{T} \\
* & -\gamma \lambda & 0 & \hat{D}_{\delta \delta_{i}}^{T} & \hat{D}_{1 \delta_{i}}^{T} \\
* & * & -\gamma I & \hat{D}_{\delta 1_{i}}^{T} & \hat{D}_{1 i_{i}}^{T} \\
* & * & * & -\gamma \lambda & 0 \\
* & * & * & * & -\gamma I
\end{array}\right]\left(\begin{array}{cc}
N_{R} & 0 \\
0 & I
\end{array}\right)<0 \\
\sum_{i=1}^{r} \alpha_{i}\left(\begin{array}{cc}
N_{S} & 0 \\
0 & I
\end{array}\right)^{T}\left[\begin{array}{ccccc}
\hat{A}_{i} S+S \hat{A}_{i}^{T} & S \hat{C}_{\delta_{i}}^{T} & S \hat{C}_{1_{i}}^{T} & \hat{B}_{\delta_{i}} & \hat{B}_{1_{i}} \\
* & -\gamma \lambda & 0 & \hat{D}_{\delta \delta_{i}}^{T} & \hat{D}_{\delta 1_{i}}^{T} \\
* & * & -\gamma I & \hat{D}_{1 \delta_{i}}^{T} & \hat{D}_{11_{i}}^{T} \\
* & * & * & -\gamma \lambda & 0 \\
* & * & * & * & -\gamma I
\end{array}\right]\left(\begin{array}{cc}
N_{S} & 0 \\
0 & I
\end{array}\right)<0
\end{gathered}
$$




$$
\left(\begin{array}{ll}
R & I \\
I & S
\end{array}\right)>0
$$

where $N_{\mathrm{R}}$ and $N_{\mathrm{S}}$ denote bases of the null spaces of $\left[\begin{array}{lll}C_{2} & D_{2 \delta} & D_{21}\end{array}\right]$ and $\left[\begin{array}{lll}B_{2}^{T} & D_{\delta 2}^{T} & D_{12}^{T}\end{array}\right]$, respectively. As the LMIs (100)(102) are sufficiently evaluated at all vertices according to Lemma 1, therefore, the LMIs (100) (102) are satisfied if the LMIs(74) $\sim(76)$ can be satisfied, for $i=1, \ldots, \mathrm{r}$.

For the error system of the disturbance observer, define a Lyapunov function $V_{o}\left(e_{\varsigma}\right)=e_{\varsigma}^{T} P e_{\varsigma}$, then

$$
\dot{V}_{o}\left(e_{\varsigma}\right)=e_{\varsigma}^{T}\left(\left(W_{d}-L_{d}\left(e_{\varsigma}\right) B_{3} V_{d}\right)^{T} P+P\left(W_{d}-L_{d}\left(e_{\varsigma}\right) B_{3} V_{d}\right) e_{\varsigma}-\left(e_{\varsigma}^{T} P B_{1}(\rho) \omega+\omega^{T} B_{1}^{T}(\rho) e_{\varsigma}\right)\right.
$$

Using Lemma 2, we have

$$
e_{\varsigma}^{T} P B_{1}(\rho) \omega+\omega^{T} B_{1}^{T}(\rho) e_{\varsigma} \leq \alpha_{1} e_{\varsigma}^{T} P B_{1}(\rho) B_{1}^{T}(\rho) P e_{\varsigma}+\alpha_{1}^{-1} \omega^{T} \omega
$$

Substitute (104) into (103), and if

$$
\dot{V}_{o}\left(e_{\varsigma}\right)<-\kappa_{o} e_{\varsigma}^{T} e_{\varsigma}-\left(\alpha_{1} e_{\varsigma}^{T} P B_{1}(\rho) B_{1}^{T}(\rho) P e_{\varsigma}+\alpha_{1}^{-1} \omega^{T} \omega\right)
$$

where $\alpha_{1}>0$, then

$$
\dot{V}_{o}\left(e_{\varsigma}\right)<-\kappa_{o} e_{\varsigma}^{T} e_{\varsigma}-\left(e_{\varsigma}^{T} P B_{1}(\rho) \omega+\omega^{T} B_{1}^{T}(\rho) e_{\varsigma}\right)
$$

holds. Recalling (94), (95) can be rewritten as

$$
\dot{V}_{o}\left(e_{\varsigma}\right)<-\kappa_{o} e_{\varsigma}^{T} e_{\varsigma}-\left(\alpha_{1} e_{\varsigma}^{T} P B_{1}(\rho) B_{1}^{T}(\rho) P e_{\varsigma}\right)-\alpha_{1}^{-1} \gamma^{-1} z^{T} z
$$

Where $\kappa_{o}$ is a small positive scalar depending on ${ }^{[32]}$

$$
\left(W_{d}-L_{d}\left(e_{\varsigma}\right) B_{3} V_{d}\right)^{T} P+P\left(W_{d}-L_{d}\left(e_{\varsigma}\right) B_{3} V_{d}\right)<0 .
$$

It further follows from (107) that

$$
\dot{V}_{o}\left(e_{\varsigma}\right)<-\left(\begin{array}{c}
z \\
e_{\varsigma}
\end{array}\right)^{T} \tilde{\Lambda}_{d}\left(\begin{array}{c}
z \\
e_{\varsigma}
\end{array}\right)
$$

where $\tilde{\Lambda}_{d}=\left[\begin{array}{cc}\alpha_{1}^{-1} \gamma^{-1} & 0 \\ 0 & \kappa_{o}+\alpha_{1} P B_{1}(\rho) B_{1}^{T}(\rho) P\end{array}\right]$. 
The closed-loop system under the DO-LPV composite controller is exponentially stable in the sense that for an initial state $x$ and $\varsigma$ satisfying $\|x(0)\| \leq \bar{x}_{0},\|\varsigma(0)\| \leq \bar{\varsigma}_{0}$, where $\bar{x}_{0}$ and $\bar{\varsigma}_{0}$ are given scalars, $\lim _{t \rightarrow \infty} x(t) \rightarrow 0$ and $\lim _{t \rightarrow \infty} e_{\varsigma}(t) \rightarrow 0$.

When RBF neural network approximation error $\varepsilon$ is considered, a candidate Lyapunov function is chosen as follows,

$$
V=x^{T} P x+V_{o}\left(e_{\varsigma}\right)+\frac{1}{2} \frac{1}{\gamma_{W}} W^{T} P W
$$

And the first equation of system (46) can be described as

$$
\dot{x}=A(\rho) x(t)+B_{1}(\rho) \omega(t)+\left(B_{2}(\rho)\left(u_{L P V}+u_{d}\right)+\Delta \Sigma+B_{3} d(t)\right)
$$

So the derivative Lyapunov function $\dot{V}$ is

$$
\begin{aligned}
\dot{V}= & x^{T} P \dot{x}+\frac{1}{\gamma_{W}} W^{T} P \dot{W}+\dot{V}_{o}\left(e_{\varsigma}\right)=x^{T} P(\Xi+\Delta \Sigma)+\frac{1}{\gamma_{W}} W^{T} P \dot{W}+\dot{V}_{o}\left(e_{\varsigma}\right) \\
& =x^{T} P \Xi+x^{T} P\left(W^{* T} h\left(x_{N N}\right)+\varepsilon\left(x_{N N}\right)\right)+\frac{1}{\gamma_{W}} W^{T} P \dot{W}+\dot{V}_{o}\left(e_{\varsigma}\right) \\
& =x^{T}\left(A_{c l}^{T}(\rho) P+P A_{c l}(\rho)+\gamma^{-2} P B_{c l}(\rho) B_{c l}^{T} P+\left[\begin{array}{c}
C_{c l}^{T} \\
D_{c l}^{T}
\end{array}\right]\left[\begin{array}{ll}
C_{c l} & D_{c l}
\end{array}\right]\right) x+x^{T} P\left(W^{* T} h\left(x_{N N}\right)+\varepsilon\left(x_{N N}\right)\right)+\frac{1}{\gamma_{W}} W^{T} P \dot{W}+\dot{V}_{o}\left(e_{\varsigma}\right) \\
& <-\kappa_{x} x^{T} x+x^{T} P \varepsilon+\left(x^{T} P W^{* T} h+\frac{1}{\gamma_{W}} W^{T} P \dot{W}\right)-\left(\begin{array}{c}
z \\
e_{\varsigma}
\end{array}\right)^{T} \tilde{\Lambda}_{d}\left(\begin{array}{c}
z \\
e_{\varsigma}
\end{array}\right)
\end{aligned}
$$

where

$$
\Xi=A(\rho) x(t)+B_{1}(\rho) \omega(t)+\left(B_{2}(\rho)\left(u_{L P V}+u_{d}\right)+B_{3} d(t)\right)
$$

$\kappa_{x}$ is a small positive scalar depending on $(20)^{[32]}$. Choose the update law of the $\mathrm{NN}$ weight function as:

$$
\dot{W}=-\gamma_{W} x^{T} h
$$

Denotes $\bar{c}_{N N}=\max \left(\left\|x^{T} P \varepsilon\right\|\right)$, and let $c_{k}=\min \left\{2 K_{x}, 2 \lambda_{\min }\left(\tilde{\Lambda}_{d}\right)\right\}$, hence, we obtain $\dot{V} \leq-c_{k} V+\bar{c}_{N N}$, according to LaSalleYoshizawa Lemma and Ref [33], the closed-loop system tracking error will exponentially converge.

\section{REFERENCES}

[1] Wildschek A.. Flight dynamics and control related challenges for design of a commercial blended wing body aircraft, In Proc. AIAA Guid., Navigat., Control Conf., AIAA SciTech Forum, National Harbor, Maryland, pp.1-12, Jan 2014

[2] Xu X. P., Zhou Z.. Study on longitudinal stability improvement of flying wing aircraft based on synthetic jet flow control. Aerospace Sci. Tech., vol.46, pp. 287-298, Aug. 2015. 
[3] Kozek M., Schirrer A., Modeling and Control for a Blended Wing Body Aircraft-A Case Study, Springer, Switzerland, 2015

[4] Castro H.V.. Flying and handling Qualities of a Fly by Wire Blended Wing Body Civil Transport Aircraft. PhD thesis, Cranfield University, Cranfield, UK, 2003.

[5] Rahman N. U., Whidborne J.F., Propulsion and flight controls integration for a blended-wing-body transport aircraft, J. Aircraft. vol.47, no.3, pp.895-903, May 2010

[6] Yann D., Joël B., Daniel A., Toussaint C. and Taquin G., Multi-control surface optimization for blended wing-body under handling quality constraints. J. Aircraft. vol.55, no.2, pp. 638-651, Sep. 2017

[7] Hoffmann C. and Werner H.. A survey of linear parameter-varying control applications validated by experiments or high-fidelity simulations, IEEE Trans. Control Syst. Technol., vol. 23, no. 2, pp. 416-433,Mar. 2015.

[8] Liu S. Q., Sang Y. J., Jin H. B., Robust model predictive control for stratospheric airships using LPV design, Control Eng. Pract., vol.81, pp. 231-243, Dec. 2018

[9] Shin J. Y., Balas G. J., and Kaya M. A., Blending methodology of linear parameter varying control synthesis of F-16 aircraft system. J. of Guid., Control, Dyn., vol.25, no.6, pp.1040-1048, Nov. 2002.

[10] Zhao P., Nagamune R.. Switching LPV controller design under uncertain scheduling parameters, Automatica No,76, pp. 243-250. 2017.

[11] Gahinet P., Apkarian P., and Chilali M., Affine parameter-dependent Lyapunov functions and real parametric uncertainty, IEEE Trans. Autom. Control. vol.41, pp. 436-442, Mar. 1996

[12] Sato M., Robust gain-scheduled flight controller for an in-flight simulator, IEEE Trans. Aerosp. Electron. syst, vol. 56, no. 3 pp. $2122-2135$, Jan. 2019.

[13] Baranyi P.. TP model transformation as a manipulation tool for QLPV analysis and design, Asian J. Control, vol.17, no.2, pp. 497-507, Mar. 2015

[14] Apkarian P., Gahinet P., and Becker G., Self-scheduled $H_{\infty}$ control of linear parameter-varying systems: a design example, Automatica, vol.31, no.9, pp.1251-1261, Sep. 1995

[15] Chumalee S. Whidborne, J. F., Gain-scheduled $H_{\infty}$ control via parameter-dependent Lyapunov functions, Int. J. Syst. Sci., vol.46, no.1, pp.125-138, 2015

[16] Wu F., Packard A., and Balas G.. LPV control design for pitch-axis missile autopilots, In Proc. the 34th Conf. Decision and Cont., New Orleans, LA,USA, pp. 188-193, 1995

[17] PfiferH., Seiler P., Robustness analysis of linear parameter varying systems using integral quadratic constraints, Int. J.of Robust Nonlinear Control, vol.25, no.15, pp. 2843-2864, Jul. 2015

[18] Chen W. H., Yang J., Guo L., and Li S.H., Disturbance-observer-based control and related methods — an overview, IEEE Trans. Indust. Electron., vol.63, no.2, pp. 1083-1095, Feb.2016

[19] Sun L., Huo W., Jiao Z. X., Disturbance-observer-based robust relative pose control for spacecraft rendezvous and proximity operations under input saturation, IEEE Trans. Aerosp. Electron. syst, vol. 54, no. 4 pp. 1605-1617, Aug. 2018

[20] Liu C.J., and Chen W. H., Disturbance Rejection Flight Control for Small Fixed-Wing Unmanned Aerial Vehicles, J. of Guid., Control, Dyn. vol.39, no.12, pp. 2804-2813. Dec. 2016.

[21] Li S.H., Yang J., Chen W. H. , Chen X.S., Disturbance Observer-Based Control-Methods and Applications, CRC press, Taylor \& Francis Group, ch14, pp.233-300, 2014

[22] Gu D.W., Petkov P. H., and Konstantinov M. M.. Robust Control Design with Matlab. Springer press, Verlag London, pp. 44-45, 2005

[23] Pellanda P. C., Apkarian P., and Tuan H. D., Missile autopilot design via a multi-channel LFT/LPV control method. Int. J. Robust Nonlinear Control, vol.12, pp.1-20, 2002

[24] Chumalee S. and Whidborne J. F., Gain-scheduled $H_{\infty}$ control for tensor product type polytopic plants, Asian J. Control, vol.17, no.2, pp. 417-431, Mar. 2015

[25] Apkarian P. and Gahinet P.. A convex characterization of gain-scheduled $H_{\infty}$ controllers. IEEE Trans. Autom. Control, vol.40, no.5, pp.853-864, 1995

[26] Gahinet P. and Apkarian P.. A linear matrix inequality approach to $H_{\infty}$ control. Int. J. Robust Nonlinear Control, No.4:421-448, 1994

[27] Herrmann G., Turner M. C., and Postlethwaite I.. Linear matrix inequalities in control. Mathematical Methods for Robust and Nonlinear Control. SpringerVerlag, Berlin Heidelberg, pp.123-142, 2007.

[28] Moore D., McCabe G., and Craig B. A.. Introduction to the Practice of Statistics, 8 Ed., W. H. Freeman and Co., New York, 2014. pp.256-269. Chap.2.

[29] Chumalee S., Robust gain-scheduled $H_{\infty}$ control for unmanned aerial vehicles, PhD thesis, Cranfield University, Cranfield, UK, 2010, pp.115-118

[30] Zhou K., Doyle J. C., and Glover K.. Robust and Optimal Control. Englewood Clis, NJ: Prentice-Hall, 1995.

[31] Gahinet P., Apkarian P., and Chilali M.. Affine parameter-dependent Lyapunov functions and real parametric uncertainty. IEEE Trans. Autom. Control, vol.41, no.3,pp.436-442, Mar.1996

[32] Chen W. H., Yang J., and Zhao Z. H., Robust control of uncertain nonlinear systems: a nonlinear DOBC approach. Journal of Dynamic Systems, Measurement and Control. Vol.138, No.7, 071002, pp. 1 9, 2016 
[33] Jin X., Adaptive fault tolerant control for a class of input and state constrained MIMO nonlinear systems', International Journal of Robust \& Nonlinear Control, No.26, pp. 286-302, 2016.

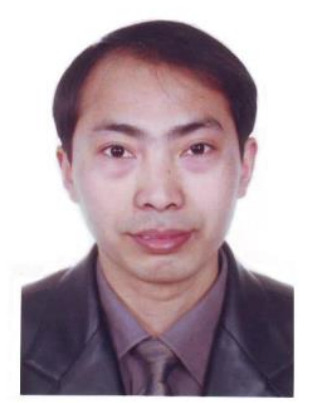

Shiqian Liu received the B.S. degree from the Department of Mechanic Engineering in 1996 in Xi'an Shiyou University, and MSc and PhD in Control Engineering from Nanjing University of Science and Technology (NUST), China. From 2005 to 2007 he was a postdoctoral research fellow in the national Key lab of Intelligent Technology and System, Tsinghua University. From 2019 to 2020 he is a visiting scholar in the School of Aerospace, Transport and Manufacturing, Cranfield University, United Kingdom. He is currently an associate professor in the School of Aeronautics and Astronautics, Shanghai Jiao Tong University. He has about 60 refereed research publications, including one book. His research interests are in the nonlinear control systems, linear parameter varying systems, fault tolerant control, airship guidance and control, UAV control, as well as aircraft modelling and simulation.

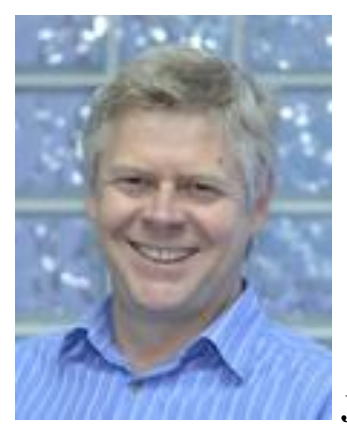

James F. Whidborne received his BA in engineering from the Cambridge University, United Kingdom, and MSc and PhD in systems and control from the University of Manchester Institute of Science and Technology (UMIST), United Kingdom. From 1991 to 1994, he held a position of Research Associate with the Department of Engineering, University of Leicester, United Kingdom. From 1994 to 2003, he was a Lecturer, then Senior Lecturer with the Department of Mechanical Engineering, Kings College London. He is currently the Head of the Dynamics Simulation and Control Group in the Department of Aerospace Engineering at Cranfield University, United Kingdom. He has about 180 refereed research publications, including three books. He is a Chartered Engineer, a Member of the IET and a Senior Member of the IEEE. His research interests are in the theory and application of advanced control, including multiobjective robust control, fluid flow control, finite precision controller implementation problems and flight control problems.

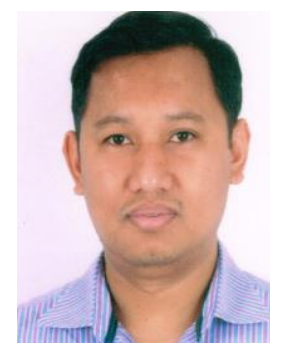

Sunan Chumalee obtained his B.Eng.,M.Sc., and Ph.D. degrees from Cranfield University, UK, in 1995, 1997, and 2011, respectively. He is currently Chief of the Space Technology Section of the Research and Development Centre for Space and Aeronautical Science and Technology, Royal Thai Air Force, Thailand. His research interests include aircraft parameter identification techniques and linear parameter varying controller design for unmanned aerial vehicles. 
2021-03-24

\title{
Disturbance observer enhanced neural network LPV control for a blended-wing-body large aircraft
}

\author{
Liu, Shiqian
}

IEEE

Liu S, Whidborne JF, Chumalee S. (2021) Disturbance observer enhanced neural network LPV control for a blended-wing-body large aircraft. IEEE Transactions on Aerospace and Electronic Systems, Volume 57, Number 5, October 2021, pp. 2689-2703

https://doi.org/10.1109/TAES.2021.3068429

Downloaded from Cranfield Library Services E-Repository 